Published in final edited form as:

ACS Appl Mater Interfaces. 2019 June 12; 11(23): 20615-20627. doi:10.1021/acsami.9b02927.

\title{
Biocompatible Carbon Nanotube-based Hybrid Microfiber for Implantable Electrochemical Actuator and Flexible Electronic Applications
}

\author{
Ting Zheng ${ }^{a, b, c, \neq}$, Parisa Pour Shahid Saeed Abadia,b,d,, , Jungmok Seo ${ }^{a, b, e, \neq}$, Byung-Hyun \\ Cha $^{a, b, f}$, Beatrice Miccoli ${ }^{a, b, g}$, Yi-Chen Li ${ }^{a, b}$, Kijun Park ${ }^{e}$, Sunghyun Park ${ }^{\dagger}$, Seon-Ji Choi ${ }^{a, b}$, \\ Rasoul Bayaniahangar ${ }^{d}$, Dongxing Zhang ${ }^{c}$, Soo-Hong Lee ${ }^{f}$, Chang-Kee Lee ${ }^{h}$, Ali

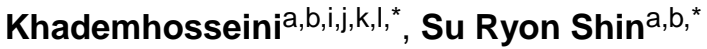 \\ aDivision of Engineering in Medicine, Department of Medicine, Brigham Women's Hospital, \\ Harvard Medical School, Cambridge, Massachusetts, 02139, USA. \\ bHarvard-MIT Division of Health Sciences and Technology, Massachusetts Institute of Technology, \\ Cambridge, Massachusetts 02139, USA. \\ 'School of Materials Science and Engineering, Harbin Institute of Technology, 150001, China \\ dDepartment of Mechanical Engineering-Engineering Mechanics, Michigan Technological \\ University, 1400 Townsend Drive, Houghton, Michigan 49931, USA. \\ eSchool of Electrical and Electronic Engineering, Yonsei University, Seoul, 03722, Republic of \\ Korea. \\ fDepartment of Biomedical Science, CHA University, 13488, Republic of Korea. \\ 9Department of Electronics and Telecommunications, Politecnico di Torino, Torino, 10129, Italy. \\ ${ }^{\text {h} K o r e a ~ P a c k a g i n g ~ C e n t e r, ~ K o r e a ~ I n s t i t u t e ~ o f ~ I n d u s t r i a l ~ T e c h n o l o g y, ~ B u c h e o n, ~ G y e o n g g i-d o ~ 14449, ~}$ \\ South Korea.
}

\footnotetext{
*Corresponding Author khademh@ucla.edu (Ali Khademhosseini), sshin4@bwh.harvard.edu (Su Ryon Shin).

¥These authors contributed equally to this work.

*These authors contributed equally as corresponding authors to this work.

Author Contributions

The manuscript was written through contributions of all authors. All authors have given approval to the final version of the manuscript. ASSOCIATED CONTENT

Supporting Information.

The Supporting Information is available free of charge on the ACS Publications website at DOI:

The SEM and TEM images of well dispersed HA/SWCNT solutions with $7 \mathrm{mg} / \mathrm{mL}$ SWCNTs; Chemical modification sites in HA and the schematic representations of HA crosslinked with HMDA after activation using EDC and HOBt; FTIR spectra of SWCNTs, uncrosslinked and crosslinked HA/SWCNT microfibers; 3D topography of uncrosslinked and crosslinked HA/CNTs microfiebrs with $7 \mathrm{mg} / \mathrm{mL}$ SWCNT concentration obtained using AFM; Tensile strength of uncrosslinked and crosslinked swollen HA/SWCNT microfibers as a function of SWCNT concentration; Failure strain of uncrosslinked and crosslinked swollen HA/SWCNT microfibers as a function of SWCNT concentration; SEM images of well dispersed HA/SWCNT solutions with $4 \mathrm{mg} / \mathrm{mL}$ and $7 \mathrm{mg} / \mathrm{mL}$ SWCNTs; Micrographs and SEM of HA/SWCNT dispersion with different SWCNT concentrations; Schematic illustration of the HA/SWCNT microfibers on the paper frame with different bending angles; Schematic illustration of the specimen (bottom), photographs of the fibers being tested in a tensile tester at rest (left) and maximum strain (right), and the corresponding load-displacement curve (middle); Cyclic voltammetry outcome of the HA/SWCNT microfibers with $7 \mathrm{mg} / \mathrm{mL}$ SWCNT concentration: current vs. scan rate; Viability of NIH-3T3 fibroblasts after five days of culture on the surface of HA/SWCNT microfibers with $7 \mathrm{mg} / \mathrm{mL}$ SWCNT concentration; 
iDepartment of Bioengineering, Department of Chemical and Biomolecular Engineering, Henry Samueli School of Engineering and Applied Sciences, University of California-Los Angeles, Los Angeles, CA 90095, USA.

'California NanoSystems Institute (CNSI), University of California-Los Angeles, Los Angeles, CA 90095, USA.

kDepartment of Bioindustrial Technologies, College of Animal Bioscience and Technology, Konkuk University, Hwayang-dong, Gwangjin-gu, Seoul 05029, Republic of Korea.

'Center of Nanotechnology, Department of Physics, King Abdulaziz University, Jeddah 21569, Saudi Arabia.

\section{Abstract}

Biocompatible, electrically conductive microfibers with superior mechanical properties have received great attention due to their potential applications in various biomedical applications such as implantable medical devices, biosensors, artificial muscles, and microactuators. Here, we developed an electrically conductive and mechanically stable carbon nanotube-based microactuator with low degradability that makes it usable for an implantable device in the body or biological environments. The microfiber was composed of hyaluronic acid (HA) hydrogel and single-wall carbon nanotubes (SWCNTs) (HA/SWCNT). HA hydrogel acts as bio-surfactant and ion-conducting binder to improve the dispersion of SWCNTs resulting in enhanced electrical and mechanical properties of the hybrid microfiber. In addition, HA was crosslinked to prevent leaking of the nanotubes from the composite. Crosslinking of HA hydrogel significantly enhances Young's modulus, failure strain, toughness, stability of electrical conductivity, and resistance to biodegradation and creep of hybrid microfibers. The obtained crosslinked HA/SWCNT hybrid microfibers show excellent capacitance, and actuation behavior under mechanical loading with the low potential of $\pm 1 \mathrm{~V}$ in a biological environment. Furthermore, the HA/SWCNT microfibers exhibits excellent in vitro viability. Finally, biocompatibility is shown through the resolution of early inflammatory response in less than three weeks after implantation of the microfibers in subcutaneous tissue of mice.

\section{Graphical Abstract}

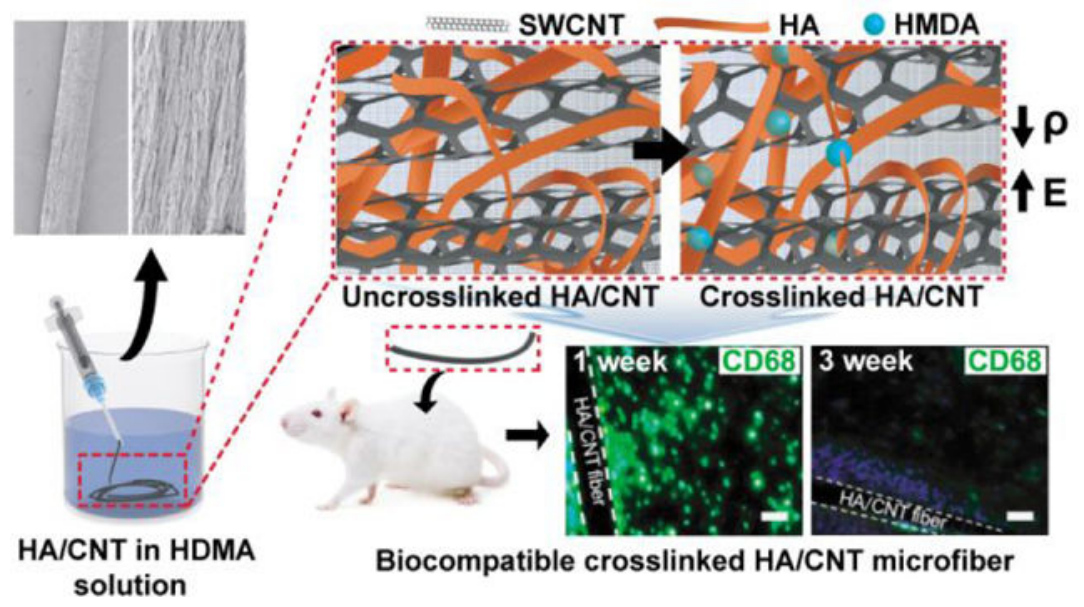




\section{Keywords}

Hyaluronic acid; Carbon nanotubes; Conductive fiber; Flexible electronics; Electrochemical microactuator; Biocompatibility

\section{Introduction}

Multifunctional microfibers have been widely researched for various applications including electronic textiles, flexible electronics, artificial muscles, implantable medical devices, and microactuators. ${ }^{1-5}$ Particularly, the development of microfiber-based actuators that can operate in the biological environment in the human body is crucial for biomedical applications such as surgical robots and actuating catheters. ${ }^{6-9}$ For the realization of microfiber-based actuators, microfibers with reliable mechanical and electrical properties are required. In addition, biocompatibility of microfibers is important for their safe operation in the body, where contact between microfibers and tissues is essential. ${ }^{10-13}$

Conventionally, ionic polymer-metal composites (IPMCs), conducting polymers, and shape memory alloys (SMAs) have been widely used to fabricate microactuators. ${ }^{14-16}$ Although IPMCs showed moderate actuation capability, cytotoxicity of the materials hinders their practical biomedical applications. ${ }^{17}$ Conducting polymers are widely used to create various types of microactuators; however, specific dopants and electrolytes with relatively high level of toxicity are required to maintain their unique electrical or electrochemical properties. SMAs are typically non-cytotoxic but suffer from low controllability at micro size, long actuator response time, and low energy efficiency. ${ }^{18}$ Recently, carbon nanotube (CNT)-based hybrid or composite materials have emerged as promising candidates for the fabrication of microactuators due to their remarkable mechanical and electrical properties in biological environments without using cytotoxic electrolytes. ${ }^{19-24}$ However, the CNT-based microfiber actuators suffer from creep, short life cycle, and low mechanical stability due to poor interactions between the assembled CNTs. When a mechanical load is applied to CNT-based microfibers, CNTs easily slide with respect to each other due to weak van der Waals interactions between the CNTs. ${ }^{25,26}$ To overcome such behavior, diverse polymeric binders have been utilized. Michardière et al. developed microfibers using polyvinyl alcohol (PVA)/CNT hybrid to increase the mechanical stiffness of microfibers. Although Young's modulus of the PVA/CNT hybrid microfibers was twice higher than that of bare CNT microfibers, stretchability under tensile strains significantly decreased. ${ }^{27}$ Similarly, Lynam et al. developed chitosan/single walled CNTs (SWCNTs) biofibers, which showed excellent tensile strength $(170 \pm 46 \mathrm{MPa})$ and elongation up to break $(6.1 \pm 0.8 \%) .{ }^{28}$ However, the electrical conductivity of chitosan/SWCNT biofibers was low $(1-2 \mathrm{~S} / \mathrm{cm})$ due to the poor electrical conductivity of chitosan. DNA/SWCNT biofibers showed improved electrical properties (conductivity: $30 \pm 4 \mathrm{~S} / \mathrm{cm}$, capacitance: $22.4 \mathrm{~F} / \mathrm{g}$ ), but the elongation of fibers up to break decreased to $3.2 \pm 0.3 \% .{ }^{29}$ Despite all the advances to date, there is a lack of highly functional microactuators with the capability to work in the human body without experiencing degradation, fatigue, or creep. 
To overcome the challenges in the way of fabrication of microactuators for use in human body, hyaluronic acid (HA) is an excellent candidate that acts as biocompatible surfactant and ion-conducting binder to improve the dispersion of SWCNTs resulting in enhanced electrical and mechanical properties of the hybrid microfiber. ${ }^{30}$ However, despite high levels of biocompatibility, HA has relatively low viscosity and mechanical stiffness. Furthermore, enzymatic reactions of hyaluronidases in the body can degrade HA. Hence the issues of creep, low fatigue resistance, and degradation of the HA/SWCNT microfibers in biological environments are unresolved. Degradation could also lead to leakage of CNTs to body during in vivo operation of the microactuators, which is not desirable. Fabrication of biocompatible microactuators with resistance to creep and degradation remains a challenge and is highly crucial for development of actuators with functionality in biological liquids for implantable and flexible electronics devices.

Here, we addressed the issues of creep, fatigue, degradability, and CNT leakage in HA/ SWCNT microactuators with developing chemically crosslinked HA/SWCNT microfibers for biocompatible electrochemical actuators. Our objectives were to design a flexible and mechanically robust SWCNT-based microfiber suitable for applications in the human body by developing a bio-surfactant and ionic-conducting binder that is stable and stiff in biological environments. The effects of SWCNT concentration and crosslinking of HA hydrogel on mechanical properties, conductivity, degradation rate, capacitance, and microactuation behavior of HA/SWCNT hybrid microfibers were comprehensively investigated. Finally, we conducted in vitro cell growth experiments on the surface of the microfibers and in vivo implantation of microfibers to evaluate the cytocompatibility and biocompatibility of the microactuators.

\section{Experimental Section}

\section{Fabrication of HA/SWCNT microfibers}

HA/SWCNT microfibers were fabricated using wet spinning technique. The weight ratio of HA (Lifecore Biomedical LLC, MW: $41 \mathrm{KDa}$ - $65 \mathrm{KDa}$ ) to SWCNTs (NanoIntegris Technologies Inc.) in suspension was optimized to produce uniform and well-dispersed solutions, and 5: 8 was chosen as the optimum ratio, which is close to those reported in literature. ${ }^{31}$ HA solutions with different concentrations $(1,2.5,3.125,3.75,4.375 \mathrm{mg} / \mathrm{mL})$ were prepared by dissolving HA in de-ionized (DI) water at room temperature. Then, SWCNTs were added and dispersed in the solution for 20 minutes using a probe sonicator (Qsonica Sonicator, Newton, USA). The SWCNTs were uniformly dispersed in the HA solution (Figure S1). To obtain microfibers, the homogeneous HA/SWCNT dispersion was injected into a rotating coagulation bath $\left(5 \% \mathrm{CaCl}_{2}\right.$ in $70 \%$ ethanol, $\left.20 \mathrm{rpm}\right)$ through a 0.2 $\mathrm{mm}$ diameter syringe needle with $50 \mathrm{~mL} / \mathrm{h}$ flow rate. The coagulated HA/SWCNT microfibers were taken out from the coagulation bath, washed with ethanol, and dried in order to remove the remaining coagulating agents. To crosslink the HA hydrogel, the coagulated microfibers were immersed in hexamethylenediamine (HMDA) solution for $1 \mathrm{hr}$ and washed with ethanol. ${ }^{32}$ Then, the crosslinked microfibers were placed into an aqueous solution of 1-Ethyl-3-[3-(dimethyl amino)propyl]carbodiimide (EDC) $(5.4 \mathrm{mg} / \mathrm{mL})$ and 1hydroxybenzotriazole monohydrate $(\mathrm{HOBt})(3.8 \mathrm{mg} / \mathrm{mL})$ for 10 minutes, to activate the 
carboxyl groups within the structure of HA. To complete the reaction, HMDA was added to the solution, and the solution was then incubated at $37^{\circ} \mathrm{C}$ for $1 \mathrm{hr}$. The molar ratio of HMDA to the carboxyl group of HA was 1:1. The obtained HA/SWCNT microfibers were then dried in air.

\section{Characterization of the dispersion of HA/SWCNT suspension}

Homogeneity of the HA/SWCNT dispersion was assessed with 3D digital microscopy (DSX-CB, Olympus Ltd., Japan), scanning electron microscopy (SEM) (S-4700, Hitachi Ltd., Japan) and field emission high resolution transmission electron microscopy (HR-TEM) (Tecnai G2, FEI, USA). After ultrasonication, for optical imaging, drops of the HA/SWCNT suspension were placed on a glass slide for microscope imaging. For SEM imaging, drops were placed on SEM stubs, dried for $24 \mathrm{~h}$ in air, and sputter coated with gold for 1 minute prior to examination in SEM with an operating voltage of $10 \mathrm{kV}$. For TEM imaging, a $10 \mu \mathrm{L}$ drop of the HA/SWCNT suspension was first diluted in $10 \mathrm{~mL}$ of distilled water, then placed on a copper grid to image using $80 \mathrm{kV}$ operating voltage.

\section{Characterization of the structure of HA/SWCNT microfibers}

The morphology and microstructure of HA/SWCNT microfibers were characterized using SEM. The surface roughness of microfibers was probed by an atomic force microscope (AFM, Bruker AXS, German) in tapping mode. The Raman spectra were measured using a Raman spectrometer (XploRA, HORIBA Jobin Yvon, France) with an excitation wavelength of $532 \mathrm{~nm}$ and a laser power of $1 \mathrm{~mW}$. Fourier transformed infrared spectroscopy (FTIR, Nicolet is50, Thermo Fisher Scientific, USA) was used to confirm the functional groups present in the uncrosslinked and crosslinked HA/SWCNT microfibers. The fibers were cut into small pieces and were grinded with $\mathrm{KBr}$. Then, the powder was pressed by tablet press for $20 \mathrm{~s}$ and was tested at room temperature.

\section{Degradation testing of HA/SWCNT microfibers}

Degradation of the HA/SWCNT microfibers was tested by placing microfibers with equal weight in closed bottles containing Dulbecco's phosphate-buffered saline (DPBS) (2 mL) and hyaluronatelyase $(50 \mathrm{U})$ at $37^{\circ} \mathrm{C}$. HA digestion was monitored by measuring the absorbance values from the DPBS supernatant $(2 \mu \mathrm{L})$ at a wavelength of $260 \mathrm{~nm}$ using a plate reader (NanoDrop 1000, Thermo Scientific). Three samples for each condition were tested, and the average value of absorbance was calculated.

\section{Mechanical characterization of HA/SWCNT microfibers}

The mechanical properties of HA/SWCNT fibers were determined using a uniaxial mechanical tester (Instron, Norwood, USA) in a tensile mode. The ends of the HA/SWCNT microfibers were fixed on a $2 \mathrm{~cm}$ paper frame by applying a superglue. For wetting the dry HA/SWCNT fibers, we sprayed the fibers and allowed them to swell for 5 minutes before testing. The stress-strain curves were plotted up to the point of failure of the microfibers. Young's modulus was measured as the slope of the linear region of the stress-strain curve. Tensile strength and failure strain were determined using the maximum stress and strain at the point of failure, respectively. Toughness was calculated as the area under the stress-strain 
curve. Average values of the properties measured for at least three specimens for each sample condition were reported.

\section{Electrical characterization of HA/SWCNT microfibers}

The electrical conductivity of the HA/SWCNT microfibers was measured by four-point probe method for a minimum of three fibers for each condition. A constant current of $1 \mathrm{~mA}$ was applied through the outer electrodes using a source meter (B2900 Series, Agilent, USA) and the voltage difference was measured between the two inner electrodes after $10 \mathrm{~s}$ using a digital multimeter (34401A, Agilent, USA). Silver paste was used at the contacting points between the fiber and the electrode probes in order to eliminate contact resistance. The diameters of the microfibers were measured using microscope images and the conductivity $(\sigma)$ was calculated using Equation (1):

$$
\sigma=(\mathrm{L} \times \mathrm{I}) /(\mathrm{S} \times \mathrm{V})
$$

where $\mathrm{S}$ is the cross-sectional area of the HA/SWCNT microfibers, $\mathrm{L}$ is the length between the two inner points, $\mathrm{V}$ is the voltage across the two inner probes, and $\mathrm{I}$ is the constant current applied to the fibers.

\section{Electrochemical capacitance of HA/SWCNT microfibers}

$\mathrm{CV}$ curves were recorded to measure the capacitance of HA/SWCNT microfibers using an electrochemical workstation (Model 600E Series, CH Instruments, USA). A three-electrode electrochemical cell was used for the CV tests. The HA/SWCNT microfiber, an $\mathrm{Ag} / \mathrm{AgCl}$ electrode, and a Pt electrode were used as the working electrode, reference electrode, and counter electrode, respectively. The electrochemical capacitance of HA/SWCNT microfibers was measured from the $\mathrm{CV}$ curves obtained within the potential window of -1 to $1 \mathrm{~V}$ with $25-100 \mathrm{mV} / \mathrm{s}$ scan rate in the DPBS solution. The HA/SWCNT microfibers were held in the DPBS for 30 mins before the tests.

\section{Characterization of the actuation behavior of HA/SWCNT microfibers}

The actuation behavior of HA/SWCNT microfibers was tested by using Muscle Lever Arm System (300C-LR, Aurora Scientific Inc., Canada) combined with the electrochemical workstation. The microfibers were held vertically in the electrolyte solution; the bottom end of the microfiber firmly clamped to the electrochemical workstation by Pt wire, and the top end of the microfiber was fixed on the strain sensor in the lever arm system by silicone epoxy glue. Then the microfiber was immersed in the DPBS solution for $2 \mathrm{hrs}$ under an applied tensile force of $4 \mathrm{mN}$ to equilibrate. The applied potential changed between -1 and $+1 \mathrm{~V}$ with a scan rate of $25 \mathrm{mV} / \mathrm{s}$. The actuation strain due to expansion/contraction of the fiber was calculated using:

$$
\text { Strain }(\%)=\left(\left(\mathrm{I}-\mathrm{I}_{0}\right) / \mathrm{I}_{0}\right) \times 100
$$

where $\mathrm{I}$ and $\mathrm{I}_{0}$ represent the instantaneous and original length of the microfiber, respectively. 


\section{Evaluation of the in vitro cytocompatibility of HA/SWCNT microactuators}

To test cell compatibility, $1 \times 10^{6} \mathrm{NIH}-3 \mathrm{~T} 3$ fibroblasts were cultured on HA/SWCNT microfiber using standard cell culture protocols. Cells were seeded directly on the surface of the microfibers after sterilization of the samples using ultraviolet light. High glucose Dulbecco's Modified Eagle Medium (DMEM) supplemented with 10\% fetal bovine serum (FBS) and $1 \%$ penicillin-streptomycin was used as medium to culture cells at $37^{\circ} \mathrm{C}$ and $5 \%$ $\mathrm{CO}_{2}$. Cell viability was evaluated using a Live/Dead assay kit (calcein-AM/ethidium Bromide homodimer, Invitrogen) according to the manufacturer's instructions. The viability of cells was calculated using Image $\mathbf{J}$ software based on counting the number of live and dead cells and then dividing them by total cell number. Immunocytochemistry of the fibroblast cells was performed after 5 days of cell culture. The cells seeded on the microfibers were fixed using $4 \%$ formaldehyde for $1 \mathrm{hr}$ at room temperature, followed by incubation in $0.1 \%$ Triton X-100 for 30 minutes, and washing 3 times with the DPBS. Then, the fibers were incubated with Alexa Fluor 488-phalloidin and DAPI to stain the F-actin and cell nuclei as described in the manufacturer's instruction. The fluorescence images were captured using an inverted microscope (Nikon, Eclipse TE 2000U, Japan).

\section{In vivo implantation of HA/SWCNT microfibers for evaluation of biocompatibility}

Six-week-old, female mice (C57 BL/6, Orientbio, Seoul, Korea) were anaesthetized with xylazine and ketamine. Uncrosslinked and crosslinked HA/SWCNT microfibers were implanted into the dorsal subcutaneous spaces of mice $(n=4$ per group). After one and three weeks, the implanted HA/SWCNT microfibers were retrieved, fixed in $4 \%(\mathrm{v} / \mathrm{v})$ paraformaldehyde (PFA), embedded in paraffin, and sectioned transversely into $6-\mu \mathrm{m}$-thick sections. For histological analysis, the prepared sections were stained with Hematoxylin (Sigma-Aldrich) and Eosin (Sigma-Aldrich) staining and rinsed with distilled water for microscopy analyses of the implanted area. For immunohistochemical analysis, prepared sections were deparaffinized and hydrated by sequential incubations in xylene and ethanol. After being washed with the DPBS for 2 minutes, the sections were pre-blocked with $3 \%$ $\mathrm{H}_{2} \mathrm{O}_{2}$ for 10 minutes. Following incubation with the primary antibody against CD68 (Abcam) and CD86 (Abcam) for $1 \mathrm{hr}$ at room temperature, the sections were incubated with Alexa Fluor 488 goat anti-mouse antibody and Texas Red goat anti-rabbit antibody (Applied Biological Materials Inc). Fluorescence images were captured using a Nikon microscope Eclipse 55i (Nikon, Kanagawa, Japan).

\section{Statistical analysis}

Statistical significance was evaluated by performing one-way ANOVA tests (GraphPad Prism 5.02, GraphPad Software). To analyze and assess significant differences between selected treatments, Tukey's multiple comparison tests were utilized. Differences were characterized as significant for $* p<0.05, * * p<0.01$, and $* * * p<0.001$.

\section{Results and Discussion}

We fabricated HA/SWCNT microfibers using a motorized wet-spinning method. The motorized wet-spinning system was composed of a syringe pump for the injection of HA/ SWCNT suspension and an automatic rotator for rotation of the coagulation bath (Figure 
1a). Microfibers were produced by spinning of $\mathrm{HA} / \mathrm{SWCNT}$ suspension in $\mathrm{CaCl}_{2}$ as coagulation bath. The liquid suspension is solidified in the coagulation bath through a combination of inter- and intra-chain ionic crosslinking and solvent-induced fiber formation. Upon injection of the HA/SWCNT suspension into the $\mathrm{CaCl}_{2}$ coagulation bath, calcium ion physical crosslinking of HA chains through D-glucuronic acid residues occurs. ${ }^{30}$ After fiber formation, we took the wet-spun fibers out of the coagulation bath and dried them at room temperature with stretching forces to further align the SWCNTs along the fiber axis. The produced fibers are meter-scale long and have circular cross-sections with a constant diameter along the entire length (Figure 1b,c), which is precisely controllable by the injection rate of the ink and the rotation rate of the rotor. Using a suspension solution composed of SWCNTs and HA (1.6:1 weight ratio) in water, an injection rate of $50 \mathrm{~mL} / \mathrm{h}$ and a rotation rate of $20 \mathrm{rpm}$, the fibers had an approximate diameter of $50 \mu \mathrm{m}$ at dry state (Figure 1c-(i)). Due to the shear force in the process of solution injection and coagulation, the surface showed nanoscale roughness along the microfibers (Figure 1c-(ii)).

The orientation and alignment of SWCNTs within the HA/SWCNT microfibers were further characterized using polarized Raman spectroscopy. Evaluation of the intensity of the laser beam upon the change of the polarization axis of the electric field in the laser beam with respect to a reference axis shows the degree of anisotropy within a composite material. ${ }^{33}$ With the change of electric field axis from perpendicular $\left(90^{\circ}\right)$ to parallel $\left(0^{\circ}\right)$ to the HA/ SWCNT microfibers, the beam intensity is increased, confirming the structural anisotropy of the HA/SWCNT microfibers (Figure 1d). ${ }^{34}$ The maximum G-band intensity, which is a qualitative indication of the orientation of SWCNTs, is gradually increased as the angle between the incident beam and fiber axis is increased. The increase in the maximum G-band intensity indicates that SWCNTs are highly aligned within the fibers along with the direction of the fiber axis (Figure 1e). ${ }^{35}$

HA is a naturally-occurring biomolecule in the human body and has been widely used for various biomedical applications such as drug delivery and tissue engineering. ${ }^{36}$ However, it is highly degradable in body through the enzymatic reactions of hyaluronidases. The chemical modification of HA is a reliable way to improve its degradation performance. HA derivatives not only maintain the original characteristics such as biological compatibility and cell adhesion, but also provide a series of other excellent features such as mechanical strength, viscosity, resistance to hyaluronidase degradation and targeting characteristics. ${ }^{37}$ HA derivatives have been widely used in tissue engineering, drug delivery, wound healing, and devices in several surgical procedures. ${ }^{38,39}$ The three most commonly used chemical modification sites in $\mathrm{HA}$ are carboxylic groups, hydroxyl groups, and $-\mathrm{NHCOCH}_{3}$ groups (Figure S2). Carboxylic groups have a higher level of activity and are the receptors of HA or hyaluronidase identification points. Therefore, carboxylic groups were chosen as the target for modification to minimize the biodegradation of HA. In this paper, HMDA was used as a chemical crosslinker of HA (Figure 2a). Wet-spun fibers were placed in an aqueous solution of EDC and HOBt to activate the carboxyl groups in the backbone of HA biomolecules.

HMDA was then added to crosslink HA chains by chemical bonding between the amide and carboxyl groups. ${ }^{32}$ Figure S2 displays a schematic representation of HA crosslinked with HMDA after activation using EDC and HOBt. The crosslinking of HA in SWCNT 
microfibers was confirmed by FTIR spectroscopy, SEM, AFM, Raman, degradation test and tensile test. Comparing with SWCNTs, both uncrosslinked and crosslinked HA/SWCNT microfibers show a broad absorption peak at $3600 \mathrm{~cm}^{-1}-3000 \mathrm{~cm}^{-1}$ and absorption peaks at $3000 \mathrm{~cm}^{-1}-2800 \mathrm{~cm}^{-1}$ in Figure S3 due to the $\mathrm{O}-\mathrm{H}$ and $\mathrm{C}-\mathrm{H}$ stretching vibrations in HA, respectively. ${ }^{40}$ Several highly overlapped bands also exist in the spectrum for uncrosslinked and crosslinked HA/SWCNT microfibers. The overlapped band at $1633 \mathrm{~cm}^{-1}$ is assigned to the $\mathrm{C}=\mathrm{O}$ stretching of amide $\mathrm{I}$ band coupled with $\mathrm{N}-\mathrm{H}$ bending in the GlcNAc unit and the antisymmetrical stretching mode of the carboxylate group in the GlcA unit. ${ }^{41,42}$ The peak was shifted to $1635 \mathrm{~cm}^{-1}$ and absorption intensity increased for crosslinked HA/SWCNT microfibers due to the overlapped absorption of N-H bending in HMDA and N-H acetamide groups in HA. Moreover, the intensity of the absorption band at $1384 \mathrm{~cm}^{-1}$ - the result of overlapped C-O stretching and N-H bending vibration of Amide III band - was increased by the introduction of $\mathrm{N}-\mathrm{H}$ vibration due to the crosslinking reaction. The peak at $1405 \mathrm{~cm}^{-1}$ represents the C-O-C stretching vibration in HA/SWCNT microfibers and was slightly shifted to $1407 \mathrm{~cm}^{-1}$ after the crosslinking reaction, which is in agreement with a previous study on HA/HMDA hybrid hydrogels. ${ }^{43}$ Figure $2 b$-(i), (ii) show the SEM images of nanofibrous networks at the fractured cross sectional areas of HA/SWCNT microfibers before and after crosslinking. The cross-sectional images of the crosslinked fibers show more winding and tangled nanofibrous networks compared to the uncrosslinked fibers with straighter and less tangled ones. Furthermore, morphological profiles from AFM images revealed rougher surfaces of the crosslinked microfibers than the uncrosslinked microfibers, which is a result of the formation of networks due to HA chemical crosslinking (Figure S4). The effect of chemical crosslinking on the nanostructure of the HA/SWCNT microfibers was evaluated using Raman Spectroscopy. In a typical Raman spectrum of CNTs, the D band peak indicates the structural defect mode, which is attributed to disordered carbon (defects and amorphous carbon), while the $\mathrm{G}$ band peak indicates the stretching mode in the graphene plane. A lower $\mathrm{D} / \mathrm{G}$ peak ratio indicates that the microfiber has fewer defects and a higher degree of graphitic crystallinity. ${ }^{44}$ The D/G ratios measured for the microfibers do not show a significant difference between the uncrosslinked $(0.18 \pm 0.01)$ and crosslinked HA/ SWCNT microfibers $(0.17 \pm 0.02)$ (Figure $2 \mathrm{c}$ ). Thus, the obtained Raman spectra suggest that the HMDA treatment does not damage the nanostructure of the SWCNTs within the microfibers.

Degradation of HA hydrogel was characterized by placing the HA/SWCNT microfibers and hyaluronidase $(25 \mathrm{U} / \mathrm{mL})$ in the DPBS for 5 days. Due to the enzymatic reactions of hyaluronidase, the color of DPBS turns dark for the uncrosslinked microfibers. The dissolution of HA hydrogel was not observed in the crosslinked microfibers (Figure 2d). HA hydrogel digestion was analyzed by measuring the absorbance values of the DPBS supernatant at $260 \mathrm{~nm}$ wavelength, which is the characteristic absorption peak of SWCNTs, over $175 \mathrm{hrs}$ after hyaluronidase treatment. A significant difference was observed between the crosslinked and uncrosslinked microfibers, showing minimal degradation of HA hydrogels in the crosslinked microfibers (Figure 2e); the absorbance value in the case of supernatant taken from the vial containing uncrosslinked HA/SWCNT microfibers was 0.38 after $120 \mathrm{hrs,}$ which was an order of magnitude higher than the value in the case of crosslinked HA/SWCNT microfibers (0.03). The carboxyl groups within the structure of HA 
have known targets for hyaluronidase in the process of HA degradation. ${ }^{45,46}$ Therefore, the lower amount of degradation of the crosslinked microfibers provides evidence for the modification of carboxyl groups due to crosslinking with HMDA. The crosslinked HA/ SWCNT microfibers are expected to be more stable in the body with the presence of hyaluronidase. Furthermore, we measured mechanical properties of the microfibers before and after the HA hydrogel digestion test (Figure 2f). After the degradation test, Young's modulus and tensile strength were, respectively, on average $75 \%$ and $600 \%$ higher for the crosslinked HA/SWCNT microfibers compared to the uncrosslinked ones. The durable mechanical integrity of crosslinked fibers in the hyaluronidase-containing liquid is well matched with the application as actuators in biological settings.

Uniaxial tensile testing of swollen fibers to the point of fracture enabled comparison of the mechanical properties of HA/SWCNT microfibers with and without chemical crosslinking and as a function of SWCNT concentration (Figure 3). Figure 3a shows representative stress-strain curves of HA/SWCNT microfibers at $6 \mathrm{mg} / \mathrm{mL} \mathrm{SWCNT}$ concentration with and without chemical crosslinking. Two stages of deformation could be identified from the stress-strain curves. First, HA/SWCNT microfibers showed linear relationship between stress and strain, suggesting an initial elastic deformation during stretching. The stress in this stage is carried by both CNT and HA with reversible stretching as the dominant mode of deformation. Then, stress within the HA/SWCNT microfibers increased nonlinearly to the point of failure of the microfibers. Irreversible sliding and slippage of CNTs and HA chains are the cause of larger deformations in this stage, whereas the stress is probably carried mainly by HA chains. Failure strain - the engineering strain at the point of fracture of HA/ SWCNT microfibers - increased with chemical crosslinking of HA and with increasing the SWCNT concentration in both the uncrosslinked and crosslinked HA/SWCNT microfibers (Figure 3b). By increasing the SWCNT concentration from 4 to $8 \mathrm{mg} / \mathrm{mL}$, the failure strain increased from $2.0 \pm 0.6 \%$ to $6.1 \pm 1.6 \%$ for uncrosslinked microfibers and from $4.9 \pm 0.4 \%$ to $11.7 \pm 2.6 \%$ for crosslinked ones. The Young's modulus and tensile strength increased dramatically with both chemical crosslinking of HA and increasing the SWCNT concentration up to $7 \mathrm{mg} / \mathrm{mL}$ (Figure $3 \mathrm{c}$ and Figure S5). The Young's modulus increased from $0.5 \pm 0.1 \mathrm{GPa}$ for $4 \mathrm{mg} / \mathrm{mL}$ uncrosslinked HA/SWCNT microfibers to $1.8 \pm 0.4 \mathrm{GPa}$ for $7 \mathrm{mg} / \mathrm{mL}$ crosslinked HA/SWCNT microfibers. The improvement of mechanical properties as the result of crosslinking is related to the formation of polymeric networks through covalent bonding of HA chains, which improve load transfer between adjacent CNTs. Significantly larger forces are required for the deformation of a chemically bonded network of polymeric chains than physically crosslinked chains (Figure 3d). Furthermore, the covalently crosslinked structure undergoes higher magnitudes of elongation before break, compared to the case of looser chains (Figure 3d). Crosslinking of HA hydrogel improved the interfacial interaction between the SWCNTs and HA network, hence resulting in preferable mechanical interlocking of SWCNTs within microfibers; stress is transferred more effectively between the HA hydrogel and the SWCNTs. SWCNTs function as loadbearing components of the hybrid structure to contribute to the stiffness and strain. Therefore, the mechanical properties of the HA/SWCNT microfibers exhibited an ascending trend with an increase in SWCNT and HA concentration, due to the increase in the density of the load-bearing SWCNT components. However, when the SWCNT concentration 
increased to $8 \mathrm{mg} / \mathrm{mL}$, Young's modulus and tensile strength of crosslinked HA/SWCNT microfibers decreased to $1.0 \pm 0.5 \mathrm{GPa}$. Such a decrease may be attributed to the poor distribution of SWCNTs in the microfibers and weak interactions between SWCNT agglomerations, which reduce the resistance to deformation of HA/SWCNT microfibers. The change in Young's modulus of uncrosslinked fibers with increasing SWCNT concentration is not significant, due to the fact that SWCNTs are less effective load-bearing components of the hybrid structure in an uncrosslinked HA hydrogel than in crosslinked one, hence decreasing the influence of SWCNT concentration on Young's modulus (Figure S6). The combination of increase in failure strain, stiffness, and strength translates to tougher microfibers through chemical crosslinking; toughness increases significantly, as determined by the extent of the area under stress-strain curves (Figure 3e). The hierarchical porous structure of the microfiber experiences higher magnitude of energy absorption before break, which is an outstanding advantage for application as an actuator. In addition to the role as load-bearing constituents of the microfibers, SWCNTs play a role in the enhanced toughness by bridging the micro- and nano-scale cracks (SWCNT pull-out; Figure 3f(i), (ii)) to increase fracture toughness and improve energy absorption. No particular trend exists in the toughness vs. SWCNT concentration due to the different trends in the failure strain and Young's modulus. The highest magnitudes of toughness correspond to the SWCNT concentrations of 5 and $7 \mathrm{mg} / \mathrm{mL}$. The combination of trends observed in failure strain, Young's modulus, and toughness shows that $7 \mathrm{mg} / \mathrm{mL}$ is the SWCNT concentration resulting in optimum mechanical properties.

We characterized the electrical properties of HA/SWCNT microfibers with different SWCNT concentrations (Figure 3g), as electrical characteristics relate directly to the performance of electrochemical actuators. For the uncrosslinked HA/SWCNT microfibers, the resistivity decreased from $1.7 \times 10^{-4} \pm 5.5 \times 10^{-5} \Omega \cdot \mathrm{m}$ to $9.1 \times 10^{-5} \pm 1.2 \times 10^{-5} \mathrm{Q}-\mathrm{m}$ as SWCNT concentration varied from $4 \mathrm{mg} / \mathrm{mL}$ to $8 \mathrm{mg} / \mathrm{mL}$. The decrease of resistivity is mainly due to the formation of more electrical pathways with increasing the amount of electrically conductive SWCNTs. Crosslinking of HA hydrogel increased resistivity; the increase in resistivity by crosslinking is attributed to the addition of the insulating crosslinker - HMDA -to the network of HA-coated SWCNTs and formation of a porous structure with the networks of HA/SWCNT (Figure 2b and Figure S4) because of chemical crosslinking. Such a porous structure decreases the number of direct contact points between the SWCNTs, resulting in lower number of existing electrical conductive pathways. Notably, the magnitude of increase in resistivity was significantly higher at the lowest and the highest SWCNT concentrations. We speculate that the highest resistivity at $4 \mathrm{mg} / \mathrm{mL}$ is related to the SWCNT density (Figure S7) being lower than the percolation threshold required for the formation of current flow pathways. The second highest resistivity at $8 \mathrm{mg} / \mathrm{mL}$ is due to the SWCNT agglomerations. SEM shows the lower density at $4 \mathrm{mg} / \mathrm{mL}$, as evident from larger pores and less compact HA-wrapped SWCNTs (Figure S7). The SWCNT agglomerations are distinguishable in both optical microscopy images of HA/SWCNT solutions (Figure S8a) and SEM images of microfibers (Figure S8b). Optical microscopy images of HA/ SWCNT dispersion (Figure S8a) show no particles greater than $3 \mu \mathrm{m}$ in size in the $7 \mathrm{mg} / \mathrm{mL}$ HA/SWCNT suspension, but $8 \mathrm{mg} / \mathrm{mL}$ HA/SWCNT suspension contained visible agglomerations $(5 \mu \mathrm{m} \sim 15 \mu \mathrm{m})$. When the HA/SWCNT suspension was assembled into 
mircofibers, these agglomerations created rough surfaces of the $8 \mathrm{mg} / \mathrm{mL}$ SWCNT concentration microfibers while HA/SWCNT microfibers with $7 \mathrm{mg} / \mathrm{mL}$ SWCNT concentration had a more uniform and smoother surface (Figure S8b). Thus, we speculate that the conductivity of HA/WCNTs microfibers were adversely affected by the agglomerations. Therefore, for the crosslinked microfibers, the best electrical performance occurs between $5-7 \mathrm{mg} / \mathrm{mL}$ SWCNT concentration.

Although chemical crosslinking of HA hydrogel increases electrical resistance, the favorable mechanical properties of the crosslinked microfibers are expected to affect their electrical conductance during actuation. To investigate the change of electrical characteristics during mechanical loading, we evaluated electrical resistivity normalized by the original resistivity $\left(\mathrm{R} / \mathrm{R}_{0}\right)$ in three modes of mechanical loading: tensile, sharp bending, and cyclic folding (i.e. fatigue testing). The bending and cyclic loading resemble high strain and repeated loading during application as actuators. Microfibers were attached to a paper with the two ends of the microfibers connected to copper wires using conducting silver paste in all three tests. Stress-strain behavior and the corresponding resistivity in tensile loading revealed a nearlinear increase for both uncrosslinked and crosslinked HA/SWCNT microfibers in resistivity with strain (Figure 3h). The increase in resistivity is a consequence of tensile strain in the material, which weakens the SWCNT junction contact points and breaks some of the electrical pathways. The crosslinked HA/SWCNT microfibers were less significantly affected by strain due to stronger SWCNT junction contact points and more stable electrical pathways. The increase in resistivity is higher for the case of uncrosslinked microfibers than crosslinked ones, as measured by bending the fiber attached to a paper substrate (Figure $3 \mathrm{i}$ and Figure S9); the uncrosslinked HA/SWCNT microfibers showed, on average, 11\% increase in resistivity while the crosslinked samples showed 6\%, revealing higher stability of crosslinked HA/SWCNT microfibers in large bending angles. The stability of the electrical conductance was further examined in cyclic mechanical deformations by folding-unfolding cycles performed on the microfiber using a mechanical tester and simultaneous measurement of resistivity (Figure $3 \mathrm{j}$ and Figure S10). The resistivity of crosslinked HA/ SWCNT microfibers remained within $10 \%$ increase in magnitude over 2500 cycles and reached a plateau of approximately $14 \%$ increase at 3000 cycles. However, the resistivity of uncrosslinked HA/SWCNT microfibers was increased significantly faster during folding cycles and reached approximately $65 \%$ increase at 3000 cycles. Crosslinking produces chemically bonded HA networks and enhanced interlocking of SWCNTs and HA, which reduce the number of micro- and nano-scale cracks at high strain and during cyclic loading. The resistance to cracks enhances the electrical performance in bending and cyclic loading. The results of both sharp bending and cycling of microfibers show that crosslinked HA/ SWCNT microfibers are superb candidates for operation as actuators, which requires exposure to such severe and repeated loading conditions.

Evaluation of the electroactivity of the HA/SWCNT microfibers through cyclic voltammetry (CV) is critical for understanding their performance as electrochemical actuators. Typical cyclic voltammogram (CV curve) of the investigated HA/SWCNT microfibers in the DPBS (pH 1.4) at different rates from 25 to $100 \mathrm{mV} / \mathrm{s}$ are presented in Figure 4a. The rectangular shape of the $\mathrm{CV}$ curves reveals the absence of faradic processes and the fact that high electrical conductivity is maintained when the HA/SWCNT microfibers are stimulated by 
voltage between $-1 \mathrm{~V}$ and $+1 \mathrm{~V}$ vs $\mathrm{Ag} / \mathrm{AgCl}$ in the DPBS. Moreover, the rectangular shape of $\mathrm{CV}$ represents typical charging-discharging performance of HA/SWCNT microfibers due to the rapid response of current to changes in potential. The HA/SWCNT microfibers showed some degree of swelling after multiple scan cycles, but still maintained their structural integrity, indicating good durability of fibers. The series of CVs at different scan rates (Figure S11) enabled calculation of electrochemical capacitance as high as $16.2 \mathrm{~F} / \mathrm{g}$ for the HA/SWCNT microfibers, which is considerably higher than that of previous biomolecule-CNT hybrid fibers. ${ }^{29}$ Although crosslinking reduced electrical conductivity, it has no significant effect on capacitance (Figure 4b). As CV is dominated by the double-layer capacitance, the electrochemical capacitance is directly related to porosity and the surface area of the materials for the adsorption of ions. ${ }^{47}$ Therefore, higher resistance and larger surface area counteract each other and cause capacitance to remain unchanged with crosslinking. SWCNT concentration also did not cause a remarkable trend in capacitance, though $7 \mathrm{mg} / \mathrm{mL}$ produces microfibers with slightly higher capacitance than the other SWCNT concentrations (Figure 4b). The measured capacitance is excellent in the DPBS, for both uncrosslinked and crosslinked microfibers, compared to other biomolecule-CNT hybrid microfibers. ${ }^{28}$ Large surface area of well-distributed HA-wrapped SWCNTs is probably the most prominent factor affecting the double-layer capacitance and the resultant $\mathrm{CV}$ curves.

The actuation behavior of the HA/SWCNT microfibers with $7 \mathrm{mg} / \mathrm{mL}$ SWCNT concentration was determined during cycling voltammetry under a $4 \mathrm{mN}$ preload (Figure 4c). The HA/SWCNT microfibers expanded when they were negatively charged to $-1 \mathrm{~V}$ and contracted when they were subsequently charged positively to $+1 \mathrm{~V}$ in the DPBS during cycling voltammetry (Figure $4 c, d$ ). The final strain remained positive with reference to the undeformed initial state during the entire cycle. Carbon nanotube fibers exhibit electrochemical actuation when electrically charged in an electrolyte. Such actuations occur via two mechanisms: (1) quantum mechanics effect, which works by a change in the length of C-C bonds upon injection of electrons or holes, and (2) double layer electrostatic effect, which is due to accumulation of ions at the electrode-electrolyte interface. ${ }^{27,48,49}$ The extent of the effect of each of the deformation mechanisms in the total actuation strain depends on several factors such as electrode structure, electrolyte, and voltage range. ${ }^{27,49}$ While application of negative potential leads to electron injection to the fiber and consequently positive strain, positive potential could cause different behaviors; both expansion and contraction as the result of positive potential have been observed in the case of CNT 49,50 Here, a combination of both double layer and quantum mechanics effects appear to play a role in the actuation mechanism (Figure 4d). If the double layer was the only influential factor, the larger size of $\mathrm{Cl}^{-}$anions compared to $\mathrm{Na}^{+}$cations should have led to larger strain in the application of the positive potential and if quantum mechanics was the only effect, contraction must have occurred during positive charging. We speculate that the double layer effect did not allow for any contraction of the fiber upon injection of positive charge. In addition to the contribution of SWCNTs in the actuation behavior, HA acts like a surfactant to produce well-dispersed aqueous SWCNT solutions, and functions as an ion-conducting binder between the SWCNTs, hence improving the capacitance and actuation behavior (Figure 4d). Crosslinking dramatically enhanced the recoverability of strain upon application of positive charge; the strain of 0.05 in the DPBS upon application of $\pm 1 \mathrm{~V}$ potential was 
almost fully recovered in several cycles while residual strain in each cycle accumulated in the uncrosslinked actuator and produced a creep-like behavior (Figure 4e). Improved elasticity due to crosslinking is in agreement with the enhanced mechanical performance of microfibers especially the higher Young's modulus as shown in Figure 3.

We further investigated the actuation behavior of the crosslinked microfibers as a function of HA/SWCNT concentration in the spinning solution (Figure 4f, g). Actuation strain ranged from $0.04-0.09 \%$, with a relatively uniform trend with the change of the composition of the spinning solution. SWCNT concentration of $7 \mathrm{mg} / \mathrm{mL}$ was the only exception with the highest strain, consistent with the measured highest capacitance (Figure $4 \mathrm{~b}$ ), due to the optimum level of SWCNT concentration to produce highest electrical conductivity. Thus, the crosslinked HA/SWCNT microfibers made of spinning solutions containing $7 \mathrm{mg} / \mathrm{mL}$ SWCNT is determined as the best electromechanical actuator in the DPBS among those tested, considering the electrical conductivity, mechanical properties, and actuation behavior. While the potential scan rate of 25 and $50 \mathrm{mV} / \mathrm{s}$ resulted in similarly excellent actuation response, an increase to $100 \mathrm{mV} / \mathrm{s}$ produced a significantly lower strain, due to the limit on the speed of response of the actuators (Figure $4 \mathrm{~h}$ ). The investigation of actuation performance as a function of scan rate sheds light on the requirement of potential scan rate in the applications requiring a specified magnitude of actuation strain; a scan rate of 50 $\mathrm{mV} / \mathrm{s}$ or lower is needed for a strain of $0.06 \%$ or higher.

Tough microfibers are excellent candidates for production of macroscale yarns and sheets through textile techniques such as braiding, weaving, and knitting. Toughness is critical as it renders them resistant to tears and material failure. Macroscale fabrics made of nanobioactuators are suitable for biomedical applications such as tissue engineering and movable devices in the human body. They are also potentially applicable to supercapacitors and flexible electromechanical devices. For a proof-of-concept demonstration of fabrication of such macroscale actuators, we prepared a bundle of 6 optimally made microactuators by manual braiding (Figure 4i). Braiding did not adversely affect the actuation behavior; the yarn showed stable $0.05 \%$ strain (Figure $4 \mathrm{i}$ ), similar to the case of a single microfiber. The stiffness of the yarn, however, is expected to be proportional to the number of used fibers, which will provide the possibility of applying larger forces with the use of more fibers in a specified direction. Thus, these HA/SWCNT microfibers are capable of operating as efficient macroscale electromechanical actuators in the DPBS under low voltage due to their excellent mechanical properties, stable electrical performance, and electrochemical efficacy of the fibers.

To examine the safety of the fabricated microfibers in the body, we first investigated in vitro cytotoxicity of the crosslinked HA/SWCNT microfibers and their applicability as tissue scaffolds. NIH-3T3 fibroblast cells, which are commonly used as cells for evaluation of cytotoxicity, were cultured on the braided HA/SWCNT microfibers. Cell viability was examined by live/dead staining. The fluorescence microscopic images of Live/Dead staining at day 1 and day 5 after cells seeding (Figure 5a, Figure S12) depict populations of mostly live cells (green color) with a few dead cells (red color) on the surface of the yarns. The cells aligned along the fiber twist direction and exhibited a stretched spindle shape. The corresponding cell viability - the ratio of the number of live cells to the total number of cells 
- was calculated on day 1 and day 5 of culture using ImageJ software. Cell viability remained higher than $80 \%$ without a significant change from day 1 to day 5 (Figure 5b). To examine cell adhesion and spreading patterns of the cells, their morphology on braided microactuators was evaluated by staining of F-actin and nuclei on day 5 of culture. The fibroblasts were spread on the surface of HA/SWCNT microfibers and exhibited elongated and well-interconnected cellular shapes, indicating excellent cell-scaffold interactions (Figure 5c). Thus, the results indicate that none of SWCNTs, HA, and the used crosslinking agents was toxic to the cells. Furthermore, the HA/SWCNT microfibers formed suitable substrates to support cell adhesion, spreading, retention and growth. Cell adhesion and cellular morphology are easily affected by the surface roughness and the mechanical properties of the microfibers. ${ }^{32,51}$ For instance, the roughness an indirect indication of porosity of the HA/SWCNT microfibers, increased with crosslinking of HA (Figure S4), which is expected to improve cell-adhesion strength. In addition, mechanical properties and surface texture are amenable via textile techniques, which is undoubtedly beneficial for cells.

Finally, we tested in vivo biocompatibility of the fabricated HA/SWCNT microfibers. Histology and immunohistology imaging of tissues surrounding the uncrosslinked and crosslinked HA/SWCNT microfibers were performed at 1 and 3 weeks after implantation in the subcutaneous tissue of mice. Hematoxylin and eosin (H\&E) staining showed that a thicker tissue is formed around the crosslinked microfiber than the uncrosslinked one. Immunohistology images stained for CD68 (monocyte/macrophage, in green) and CD86 (M1 macrophage, in red) revealed that a large number of monocytes/M1 macrophages were present around crosslinked HA/SWCNT microfibers at 1 week, compared to the uncrosslinked ones. However, after 3 weeks of implantation, the number of present monocytes/M1 macrophages decreased drastically, and only a few of them were detected in both uncrosslinked and crosslinked HA/SWCNT microfibers. Biochemical and biophysical cues presented by a biomaterial significantly influence the immune cells, especially macrophages, through altering their microenvironment. ${ }^{52-54}$ Stiffness, surface roughness, and surface chemistry of the crosslinked microfibers are different from those of uncrosslinked microfibers. All these factors could elicit the more severe early inflammatory immune response to the crosslinked microfiber. If foreign material is biocompatible, the early inflammatory response should be resolved. Therefore, the immunohistology results showing scarce monocyte/M1 macrophages at 3 weeks confirm the biocompatibility of uncrosslinked and crosslinked HA/SWCNT microactuators.

\section{Conclusion}

Biocompatible, electrically conductive and tough HA/SWCNT microfibers have been successfully spun by a wet spinning method using HA as bio-surfactant and ion-conducting binder in the spinning solution. The HA/SWCNT microfibers presented excellent electrical conductivity, mechanical properties and stable actuation behavior in the DPBS solution. The HA chains wrapped around SWCNTs surfaces and separated them into individual nanotubes to enhance the charge accumulated on the interface of HA/SWCNT microfibers and the electrolyte. HA had a significant contribution to the quantum mechanical and double-layer electrostatic effects responsible for the actuation strain. In particular, the crosslinking of HA 
offered an improvement in the mechanical properties, capacitance and the actuation stability against creep. In addition, the effects of CNT concentrations on mechanical, conductivity, electrochemical properties and actuation behavior of uncrosslinked and crosslinked HA/ SWCNT microfibers were comprehensively investigated. The crosslinked HA/SWCNT microfibers with $7 \mathrm{mg} / \mathrm{mL}$ SWCNT concentration were determined to possess optimum mechanical, electrical, and actuation properties. Furthermore, we were able to weave the microfibers into bundles, which showed an actuation strain of $0.05 \%$ in the DPBS as electrolyte under $4 \mathrm{mN}$ preload. In vitro cytocompatibility and in vivo biocompatibility experiments were carried out, and showed that the fabricated microactuators are applicable in biomedical areas. Overall, the HA/SWCNT microfibers and bundles exhibit excellent mechanical properties, stable electrical conductivity, good electrochemical and actuation behavior and, hence, prove to be promising materials for implantable microactuators and flexible electronic devices in biomedical applications.

\title{
Supplementary Material
}

Refer to Web version on PubMed Central for supplementary material.

\section{ACKNOWLEDGMENT}

\begin{abstract}
The authors gratefully acknowledge funding from the National Institutes of Health (NIH) (EB024403, AR074234, EB026824), and Air Force Office of Sponsored Research under award (FA9550-15-1-0273). The research was partially supported by a micro grant from Brigham Research Institute and Center for Faculty Development and Diversity's Office for Research Careers at Brigham and Women's Hospital. S.R.S. would like to recognize and thank Brigham and Women's Hospital President Betsy Nabel, MD, and the Reny family, for the Stepping Strong Innovator Award through their generous funding. T.Z. acknowledges the China Scholarship Council (No. 201506120155) and Harbin Institute of Technology for the financial support. P.P.S.S.A. was supported by NIH grant 5T32EB016652-02, American Heart Association grant 17SDG33660925, and an American Fellowship from American Association of University Women. This work was performed in part at the Center for Nanoscale Systems (CNS), a member of the National Nanotechnology Coordinated Infrastructure Network (NNCI), which is supported by the National Science Foundation under NSF award no. 1541959. CNS is part of Harvard University.
\end{abstract}

\section{REFERENCES}

(1). Carpi F; Kornbluh R; Sommer-Larsen P; Alici G Electroactive Polymer Actuators as Artificial Muscles: Are They Ready for Bioinspired Applications? Bioinspiration andBiomimetics 2011, 6, 045006.

(2). Kong L; Chen W Carbon Nanotube and Graphene-Based Bioinspired Electrochemical Actuators. Adv. Mater. 2014, 26, 1025-1043. [PubMed: 24338697]

(3). Cai F; Chen T; Peng H All Carbon Nanotube Fiber Electrode-Based Dye-Sensitized Photovoltaic Wire. J. Mater. Chem. 2012, 22, 14856-14860.

(4). Darbari S; Abdi Y; Mohajerzadeh S Branched Carbon Nanotubes to Realize a Novel Capacitive Sensor and Actuator Device. Sensors Actuators, A Phys. 2011, 167, 389-397.

(5). Di J; Zhang X; Yong Z; Zhang Y; Li D; Li R; Li Q Carbon-Nanotube Fibers for Wearable Devices and Smart Textiles. Adv. Mater. 2016, 28, 10529-10538. [PubMed: 27432521]

(6). Mineta T; Mitsui T; Watanabe Y; Kobayashi S; Haga Y; Esashi M Batch Fabricated Flat Meandering Shape Memory Alloy Actuator for Active Catheter. Sensors Actuators, A Phys. 2001, 88, 112-120.

(7). Fang BK; Ju MS; Lin CCK A New Approach to Develop Ionic Polymer-Metal Composites (IPMC) Actuator: Fabrication and Control for Active Catheter Systems. Sensors Actuators, A Phys. 2007, 137, 321-329. 
(8). Vara H; Collazos-Castro JE Biofunctionalized Conducting Polymer/Carbon Microfiber Electrodes for Ultrasensitive Neural Recordings. ACS Appl. Mater. Interfaces 2015, 7, 27016-27026. [PubMed: 26574911]

(9). Mineta T; Mitsui T; Watanabe Y; Seiya K; Haga Y; Masayoshi E An Active Guide Wire with Shape Memory Alloy Bending Actuator Fabricated by Room Temperature Process. Sensors Actuators, A Phys. 2002, 97, 632-637.

(10). Filip J; Šefčovičová J; Tomčík P; Gemeiner P; Tkac J A Hyaluronic Acid Dispersed Carbon Nanotube Electrode Used for a Mediatorless NADH Sensing and Biosensing. Talanta 2011, 84, 355-361. [PubMed: 21376957]

(11). Shahinpoor M; Kim KJ Ionic Polymer-Metal Composites: IV. Industrial and Medical Applications. Smart Mater. Struct. 2005, 14, 197-214.

(12). Spinks GM; Shin SR; Wallace GG; Whitten PG; Kim SI; Kim SJ Mechanical Properties of Chitosan/CNT Microfibers Obtained with Improved Dispersion. Sensors Actuators, B Chem. 2006, 115, 678-684.

(13). Moulton SE; Minett AI; Murphy R; Ryan KP; McCarthy D; Coleman JN; Blau WJ; Wallace GG Biomolecules as Selective Dispersants for Carbon Nanotubes. Carbon N. Y. 2005, 43, 18791884.

(14). Mineta T; Deguchi T; Makino E; Kawashima T; Shibata T Fabrication of Cylindrical Micro Actuator by Etching of TiNiCu Shape Memory Alloy Tube. Sensors Actuators, A Phys. 2011, 165, 392-398.

(15). Gojić M; Vrsalović L; Kožuh S; Kneissl A; Anžel I; Gudić S; Kosec B; Kliškić M Electrochemical and Microstructural Study of Cu-Al-Ni Shape Memory Alloy. J. Alloys Compd. 2011, 509, 9782-9790.

(16). Viry L; Mercader C; Miaudet P; Zakri C; Derré A; Kuhn A; Maugey M; Poulin P Nanotube Fibers for Electromechanical and Shape Memory Actuators. J. Mater. Chem. 2010, 20, 3487 3495 .

(17). Takashima K; Kamamichi N; Yagi T; Asaka K; Mukai T Cytotoxicity Test and Mass Spectrometry of IPMC. Electron. Commun. Japan 2010, 93, 1-8.

(18). Nuruddin H; Kamal IM; Mansor MN; Hafid NM A Review of Shape Memory Alloy Research, Applications and Opportunities. Mater. Des. 2014, 56, 1078-1113.

(19). Zhang X; Li Q; Holesinger TG; Arendt PN; Huang J; Kirven PD; Clapp TG; DePaula RF; Liao X; Zhao Y; Zheng L, Peterson DE, Zhu Y Ultrastrong, Stiff, and Lightweight Carbon-Nanotube Fibers. Adv. Mater. 2007, 19, 4198-4201.

(20). Sun G; Zhou J; Yu F; Zhang Y; Pang JHL; Zheng L Electrochemical Capacitive Properties of CNT Fibers Spun from Vertically Aligned CNT Arrays. J. Solid State Electrochem. 2012, 16, 1775-1780.

(21). Li J; Song L; Niu Z; Cai L; Zeng Q; Zhang X; Dong H; Zhao D; Zhou W; Xie S SuperfastResponse and Ultrahigh-Power-Density Electromechanical Actuators Based on Hierarchal Carbon Nanotube Electrodes and Chitosan. Nano Lett. 2011, 11, 4636-4641. [PubMed: 21972899]

(22). Park JM; Gu GY; Wang ZJ; Kwon DJ; Shin PS; Choi JY; Lawrence DeVries K Mechanical and Electrical Properties of Electrospun CNT/PVDF Nanofiber for Micro-Actuator Applications. Adv. Compos. Mater. 2016, 25, 305-316.

(23). Shin SR; Shin C; Memic A; Shadmehr S; Miscuglio M; Jung HY; Jung SM; Bae H; Khademhosseini A; Tang X; Dokmeci MR Aligned Carbon Nanotube-Based Flexible Gel Substrates for Engineering Biohybrid Tissue Actuators. Adv. Funct. Mater. 2015, 25, 4486-4495. [PubMed: 27134620]

(24). Zheng W; Razal JM; Whitten PG; Ovalle-Robles R; Wallace GG; Baughman RH; Spinks GM Artificial Muscles Based on Polypyrrole/Carbon Nanotube Laminates. Adv. Mater. 2011, 23, 2966-2970. [PubMed: 21590813]

(25). Zhang X; Li Q Enhancement of Friction between Carbon Nanotubes : An Efficient Strategy to Strengthen Fibers. ACS Nano 2010, 4, 312-316. [PubMed: 20020757]

(26). Lu W; Zu M; Byun J; Kim B; Chou T State of the Art of Carbon Nanotube Fibers : Opportunities and Challenges. Adv. Mater. 2012, 24, 1805-1833. [PubMed: 22438092] 
(27). Michardière AS; Mateo-Mateo C; Derré A; Correa-Duarte MA; Mano N; Poulin P Carbon Nanotube Microfiber Actuators with Reduced Stress Relaxation. J. Phys. Chem. C 2016, 120, 6851-6858.

(28). Lynam C; Moulton SE; Wallace GG Carbon-Nanotube Biofibers. Adv. Mater. 2007, 19, 1244 1248.

(29). Shin SR; Lee CK; Eom TW; Lee SH; Kwon CH; So I; Kim SJ DNA-Coated MWNT Microfibers for Electrochemical Actuator. Sensors Actuators, B Chem. 2012, 162, 173-177.

(30). Razal JM; Gilmore KJ; Wallace GG Carbon Nanotube Biofiber Formation in a Polymer-Free Coagulation Bath. Adv. Funct. Mater. 2008, 18, 61-66.

(31). Moulton SE; Maugey M; Poulin P; Wallace GG Liquid Crystal Behavior of Single-Walled Carbon Nanotubes Dispersed in Biological Hyaluronic Acid Solutions. J. Am. Chem. Soc. 2007, 129, 9452-9457. [PubMed: 17622144]

(32). Yeom J; Bhang SH; Kim B; Seo MS; Hwang EJ; Cho IH; Park JK; Hahn SK Effect of CrossLinking Reagents for Hyaluronic Acid Hydrogel Dermal Fillers on Tissue Augmentation and Regeneration. Bioconjug. Chem. 2010, 12, 240-247.

(33). Anglaret E; Righi A; Righi A; Sauvajol JL; Bernier P; Vigolo B; Poulin P Raman Resonance and Orientational Order in Fibers of Single-Wall Carbon Nanotubes. Phys. Rev. B - Condens. Matter Mater. Phys. 2002, 65, 1654261-1654267.

(34). Duesberg GS; Loa I; Burghard M; Syassen K; Roth S Polarized Raman Spectroscopy on Isolated Single-Wall Carbon Nanotubes G. Phys. Rev. Lett. 2000, 85, 5436-5439. [PubMed: 11136015]

(35). Zheng L; Sun G; Zhan Z Tuning Array Morphology for High-Strength Carbon-Nanotube Fibers. Small 2010, 6, 132-137. [PubMed: 19902432]

(36). Ju E; Choi J; Kim H; Joo C; Kwang S Biomaterials Anti-Flt1 Peptide e Hyaluronate Conjugate for the Treatment of Retinal Neovascularization and Diabetic Retinopathy. Biomaterials 2011, 32, 3115-3123. [PubMed: 21277020]

(37). Khunmanee S; Jeong Y; Park H Crosslinking Method of Hyaluronic-Based Hydrogel for Biomedical Applications. J. Tissue Eng. 2016, 8, 204173141772646.

(38). Mero A; Campisi M Hyaluronic Acid Bioconjugates for the Delivery of Bioactive Molecules. Polymers (Basel). 2014, 6, 346-369.

(39). Collins MN; Birkinshaw C Hyaluronic Acid Based Scaffolds for Tissue Engineering - A Review. Carbohydr. Polym. 2013, 92, 1262-1279. [PubMed: 23399155]

(40). Servaty R; Schiller J; Binder H; Arnold K Hydration of Polymeric Components of Cartilage - An Infrared Spectroscopic Study on Hyaluronic Acid and Chondroitin Sulfate. Int. J. Biol. Macromol. 2001, 28, 121-127. [PubMed: 11164228]

(41). Dřímalová E; Velebný V; Sasinková V; Hromádková Z; Ebringerová A Degradation of Hyaluronan by Ultrasonication in Comparison to Microwave and Conventional Heating. Carbohydr. Polym. 2005, 61, 420-426.

(42). Coimbra P; Alves P; Valente TAM; Santos R; Correia IJ; Ferreira P Sodium Hyaluronate/ Chitosan Polyelectrolyte Complex Scaffolds for Dental Pulp Regeneration: Synthesis and Characterization. Int. J. Biol. Macromol. 2011, 49, 573-579. [PubMed: 21704650]

(43). Park KL; Ma W; Higaki Y; Takahara A Design and Characterization of Hybrid Hydrogels Composed of Imogolite Fibrous Nanotubular Clay and Hyaluronic Acid. Polymer (Guildf). 2016, 100, 238-243.

(44). Mayhew E; Prakash V Thermal Conductivity of High Performance Carbon Nanotube Yarn-like Fibers. J. Appl. Phys. 2014, 15, 1331-1334.

(45). Oh EJ; Kang S; Kim B; Jiang G; Cho IH; Hahn SK Control of the Molecular Degradation of Hyaluronic Acid Hydrogels for Tissue Augmentation. J. Biomed. Mater. Res. 2008, 86, 685-693.

(46). Kim J; Kim IS; Cho TH; Lee KB; Hwang SJ; Tae G; Noh I; Lee SH; Park Y; Sun K Bone Regeneration Using Hyaluronic Acid-Based Hydrogel with Bone Morphogenic Protein-2 and Human Mesenchymal Stem Cells. Biomaterials 2007, 28, 1830-1837. [PubMed: 17208295]

(47). Lee SH; Lee CK; Shin SR; Gu BK; Kim SI; Kang TMK; Kim SJ Enhanced Actuation of PPy/CNT Hybrid Fibers Using Porous Structured DNA Hydrogel. Sensors Actuators, B Chem. 2010, 145, 89-92. 
(48). Hughes M; Spinks GM Multiwalled Carbon-Nanotube Actuators. Adv. Mater. 2005, 17, 443446.

(49). Verissimo-Alves M; Koiller B; Capaz RB; Chacham H Electromechanical Effects in Carbon Nanotubes: Ab Initio and Analytical Tight-Binding Calculations. Phys. Rev. B - Condens. Matter Mater. Phys. 2003, 67, 1-4.

(50). Shin SR; Lee CK; So I; Jeon JH; Kang TM; Kee C; Kim SI; Spinks GM; Wallace GG; Kim SJ DNA-Wrapped Single-Walled Carbon Nanotube Hybrid Fibers for Supercapacitors and Artificial Muscles. Adv. Mater. 2008, 466-470.

(51). Shin SR; Bae H; Cha JM; Mun JY; Chen YC; Tekin H; Shin H; Farshchi S; Dokmeci MR; Tang S; Khademhosseini A Carbon Nanotube Reinforced Hybrid Microgels as Scaffold Materials for Cell Encapsulation. ACS Nano 2012,, 362-372. [PubMed: 22117858]

(52). Sridharan R; Cameron AR; Kelly DJ; Kearney CJ; O’Brien FJ Biomaterial Based Modulation of Macrophage Polarization: A Review and Suggested Design Principles. Mater. Today 2015, 18, 313-325.

(53). Brown BN; Ratner BD; Goodman SB; Amar S; Badylak SF Macrophage Polarization: An Opportunity for Improved Outcomes in Biomaterials and Regenerative Medicine. Biomaterials 2012, 33, 3792-3802. [PubMed: 22386919]

(54). Adutler-Lieber S; Zaretsky I; Platzman I; Deeg J; Friedman N; Spatz JP; Geiger B Engineering of Synthetic Cellular Microenvironments: Implications for Immunity. J. Autoimmun. 2014, 54, 100-111. [PubMed: 24951031] 
a)

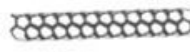

CNT

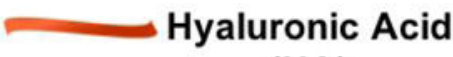

(HA)
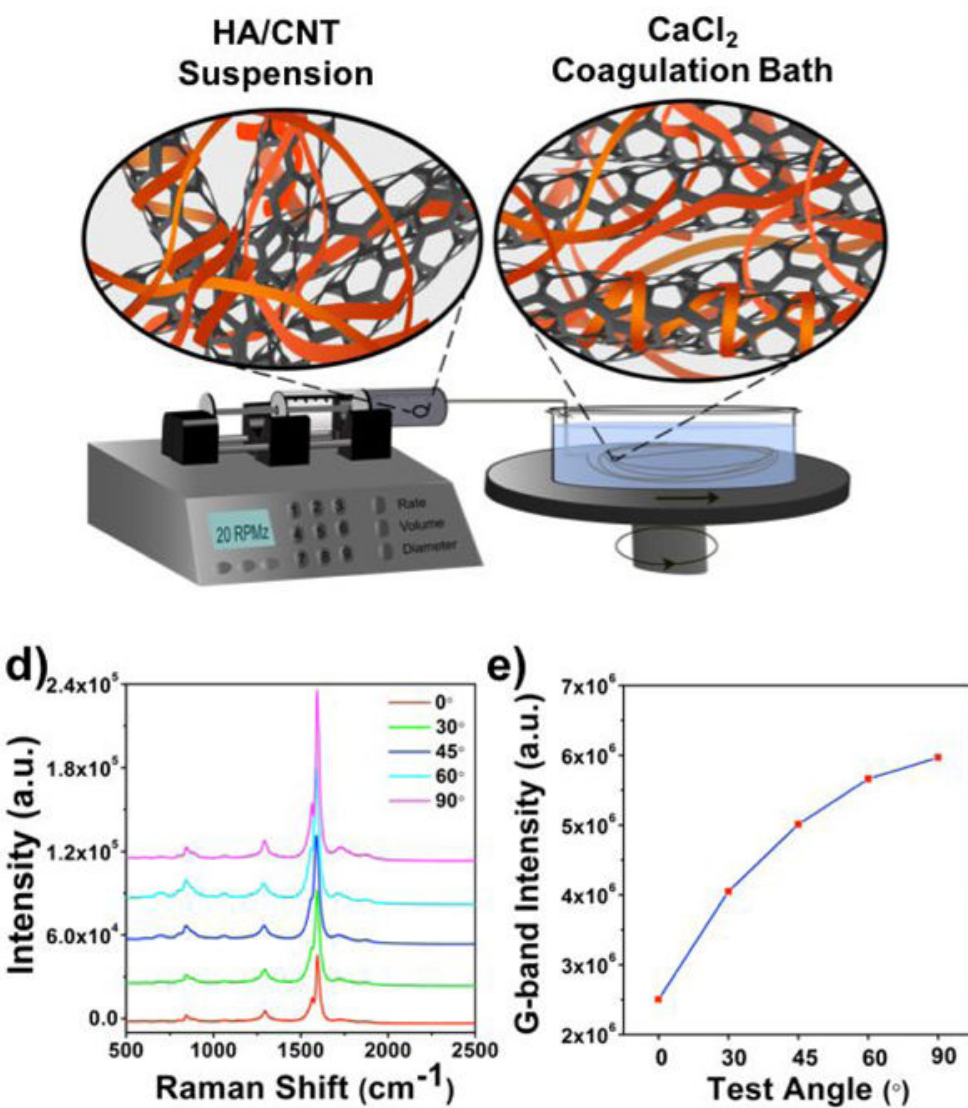

b)

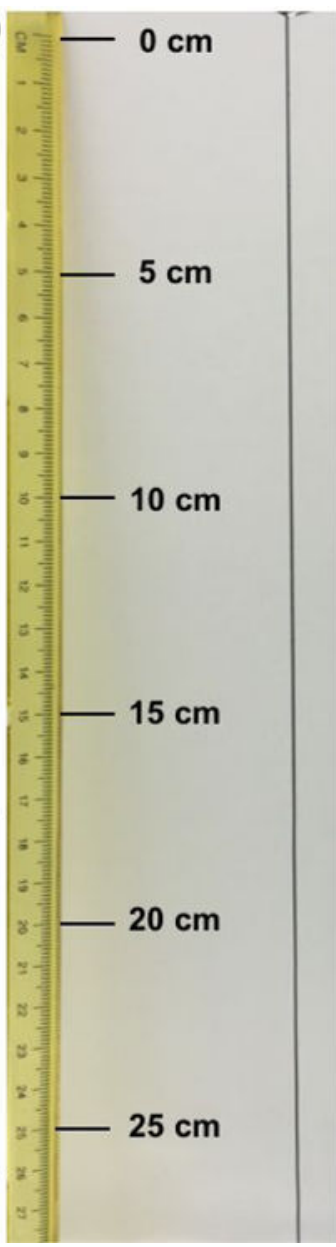

c)

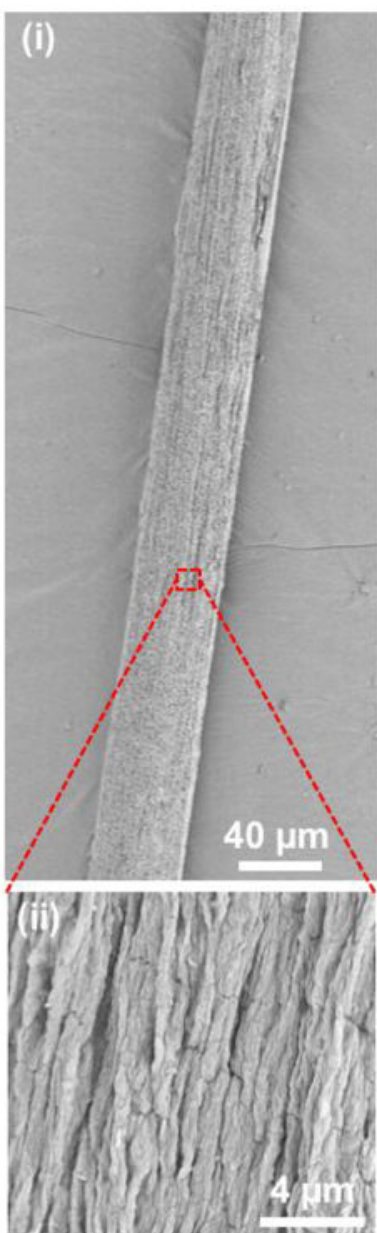

Figure 1.

a) Schematic illustration of the wet spinning set up for the fabrication of HA/SWCNT hybrid microfibers with $4 \mathrm{mg} / \mathrm{mL}$ SWCNT concentration. b) Photograph of a long HA/ SWCNT microfiber fabricated in the wet spinning process. c) SEM images of a dried HA/ SWCNT microfiber at (i) low and (ii) high magnifications. d) Polarized Raman spectra of an $\mathrm{HA} / \mathrm{SWCNT}$ microfiber with the fiber axis at $0^{\circ}, 30^{\circ}, 45^{\circ}, 60^{\circ}$ and $90^{\circ}$ with respect to the excitation polarization direction. (e) G-band peak intensities as a function of the angle between the fiber and the excitation polarization axis. 
a)
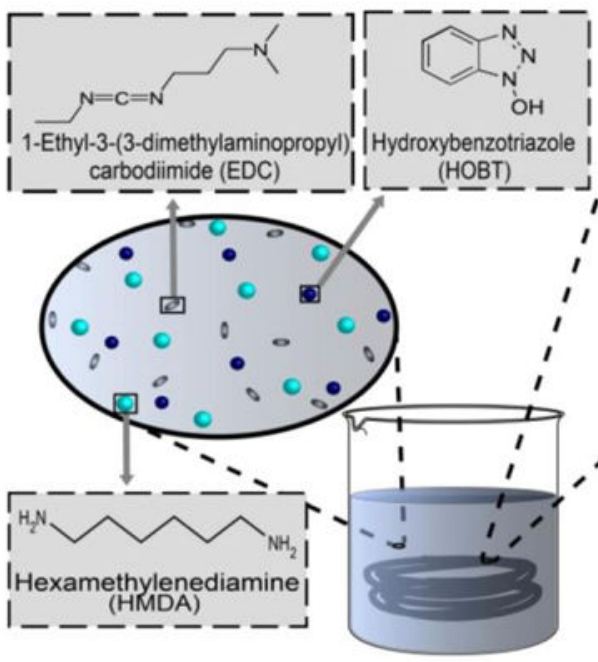

c)

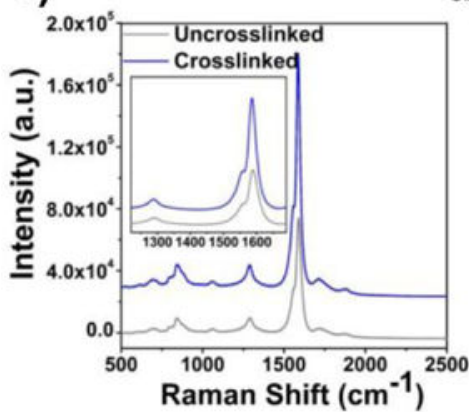

Figure 2. d)

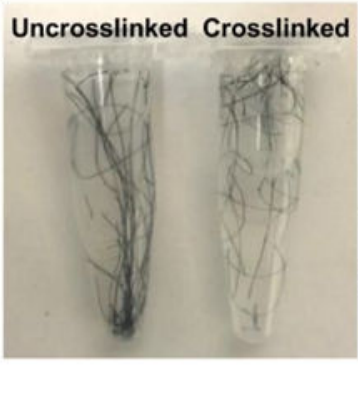

e)

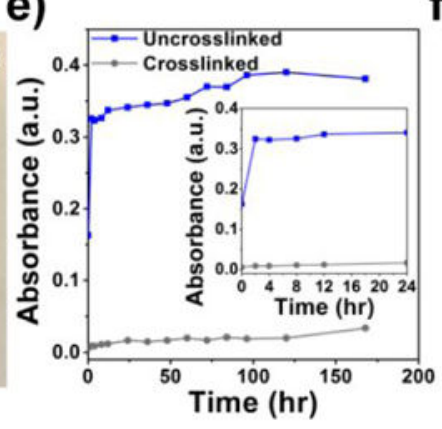

b) (i)
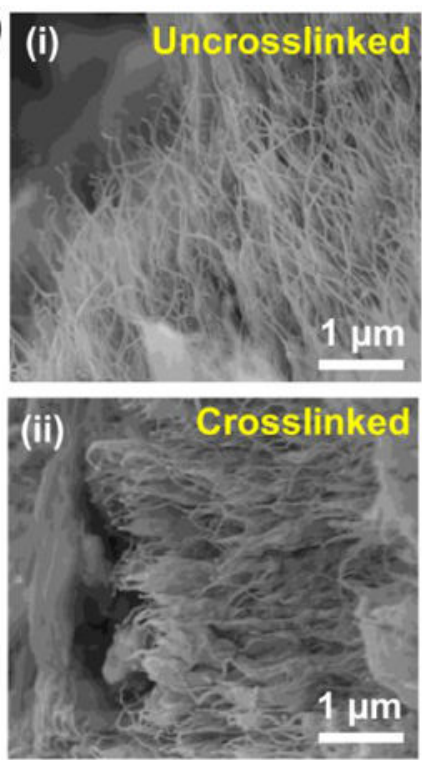

f)

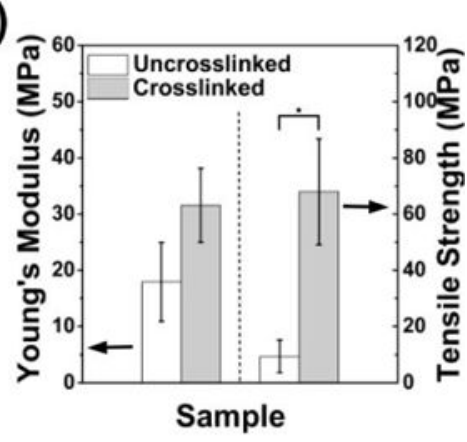

Crosslinking of HA within the HA/SWCNT microfibers with $4 \mathrm{mg} / \mathrm{mL}$ SWCNT concentration. a) Schematic illustration of the crosslinking process of HA/SWCNT microfibers. b) Cross-sectional SEM images of (i) uncrosslinked and (ii) crosslinked HA/ SWCNT microfibers. c) Polarized Raman spectra of uncrosslinked and crosslinked HA/ SWCNT microfibers. d) Photographs of HA/SWCNT microfibers in the DPBS and hyaluronidase to evaluate the degradation of HA/SWCNT microfibers. e) Absorbance at 260 $\mathrm{nm}$ measured from the DPBS supernatant containing HA/SWCNT microfibers and hyaluronidase. f) Tensile strength and Young's modulus of crosslinked and uncrosslinked HA/SWCNT fibers after 5 days storage in the DPBS and hyaluronidase. Results are presented as means \pm standard deviations $(\mathrm{n}=3 ; * \mathrm{p}<0.05, * * \mathrm{p}<0.01$, and $* * * \mathrm{p}<0.001)$. 
a)

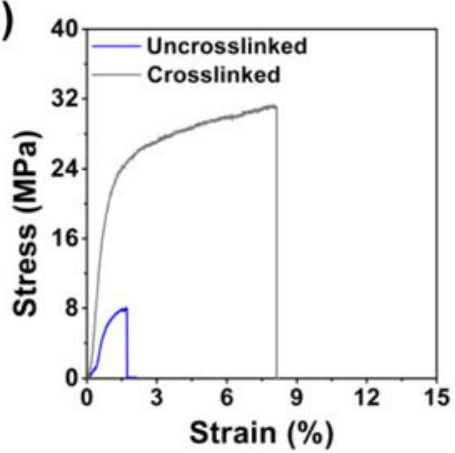

d)

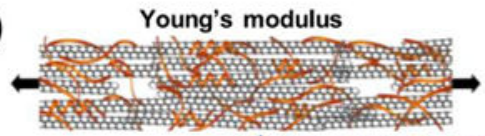

b)

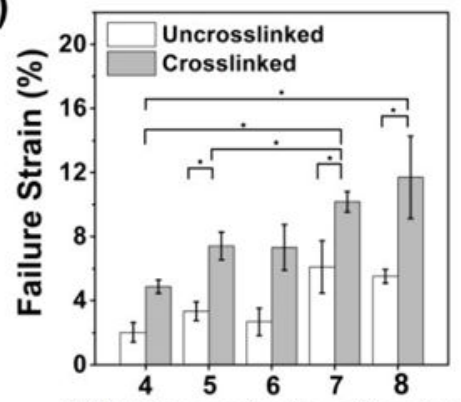

CNT Concentration $(\mathrm{mg} / \mathrm{mL}$ )

c)

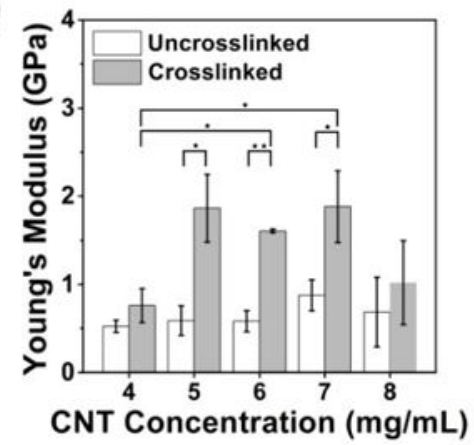

CNT Concentration $(\mathrm{mg} / \mathrm{mL}$ )

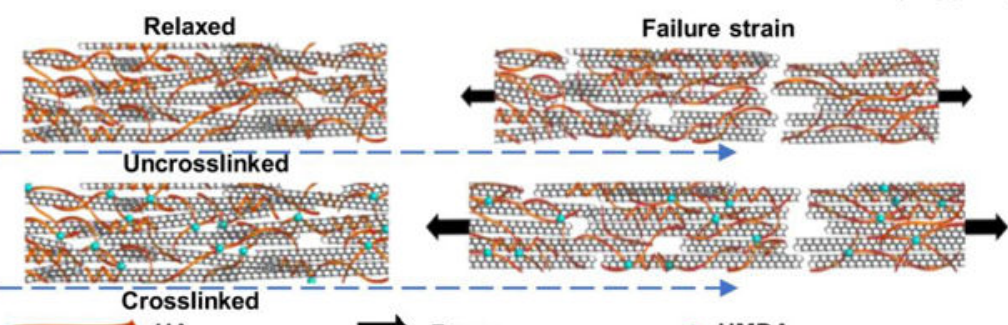

HA

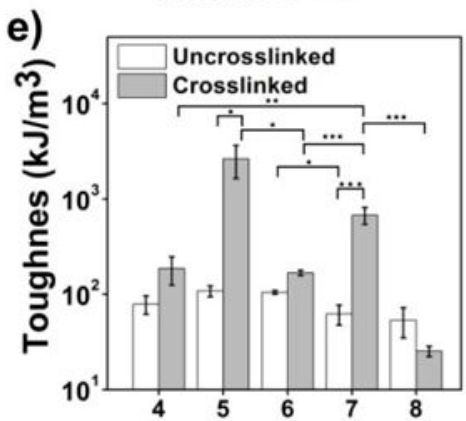

h) CNT Concentration ( $\mathrm{mg} / \mathrm{mL}$ )

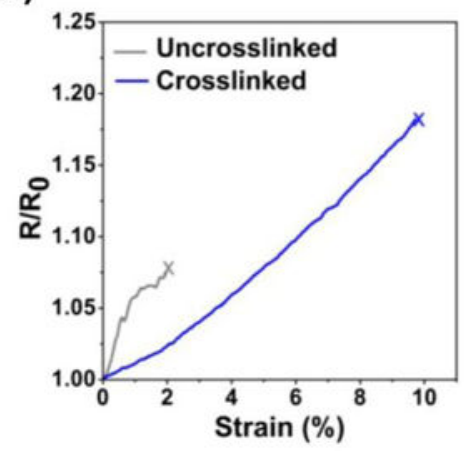

f)

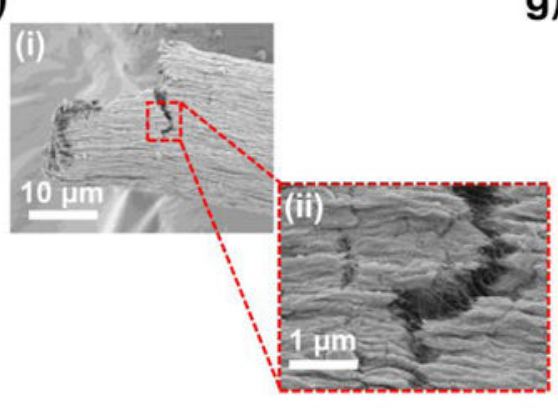

i)

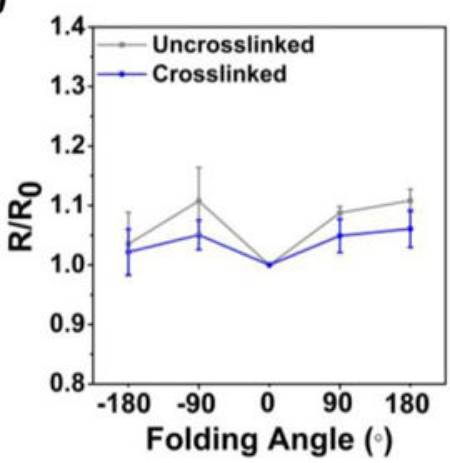

g)

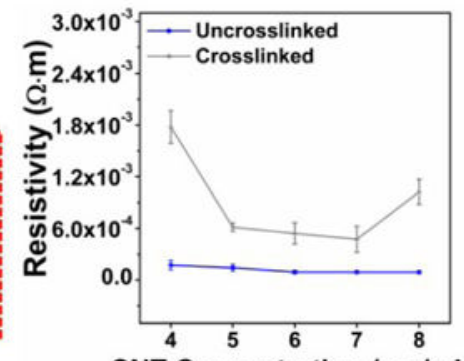

j)

CNT Concentration $(\mathrm{mg} / \mathrm{mL})$

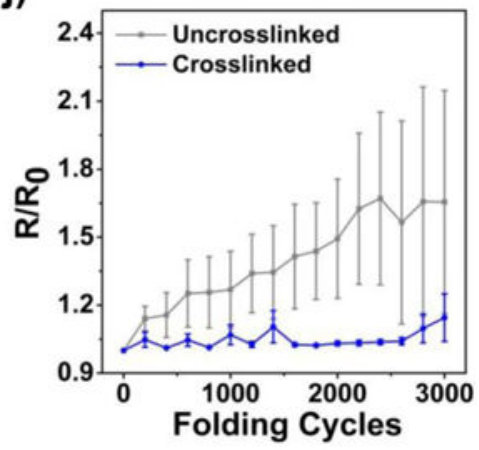

Figure 3.

Mechanical and electrical properties of HA/SWCNT microfibers. a) Stress-strain curves at 6 $\mathrm{mg} / \mathrm{mL}$ SWCNT concentration, b) Failure strain, and c) Young's modulus of uncrosslinked and crosslinked swollen HA/SWCNT microfibers as a function of SWCNT concentration. d) Schematic illustration of the mechanism of deformation of HA/SWCNT microfibers under tensile stress for uncrosslinked (top row) and crosslinked (bottom row) cases in the linear region for measurement of Young's modulus (left) and up to the point of fracture for measurement of failure strain (right). e) Toughness of uncrosslinked and crosslinked HA/ 
SWCNT microfibers as a function of SWCNT concentration. f) SEM images of the fractured cross-section of an HA/SWCNT microfiber with $1 \mathrm{mg} / \mathrm{mL}$ SWCNT concentration after tensile testing under wet condition at (i) low and (ii) high magnifications. g) Resistivity of uncrosslinked and crosslinked HA/SWCNT microfibers with different SWCNT concentrations. h) Electrical resistance of HA/SWCNT microfibers with $1 \mathrm{mg} / \mathrm{mL}$ SWCNT concentration normalized to the original resistance under uniaxial strain. i) Effect of bending on the resistance of HA/SWCNT microfibers with $1 \mathrm{mg} / \mathrm{mL}$ SWCNT concentration.. j) Stability of the electrical resistance of the HA/SWCNT microfibers with $1 \mathrm{mg} / \mathrm{mL}$ SWCNT concentration under 3000 folding-unfolding cycles. Results are presented as means \pm standard deviations $(\mathrm{n}=3 ; * p<0.05, * * p<0.01$, and $* * * p<0.001)$. 
a)

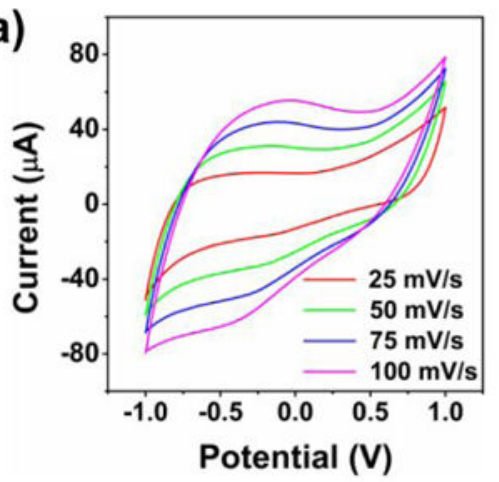

d)
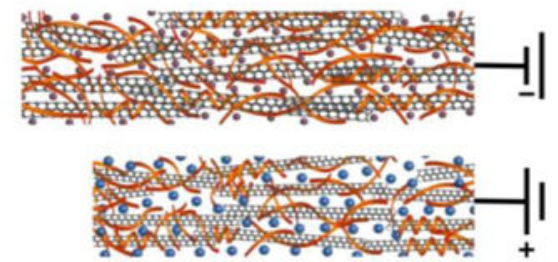

एक

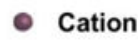

HA

g)

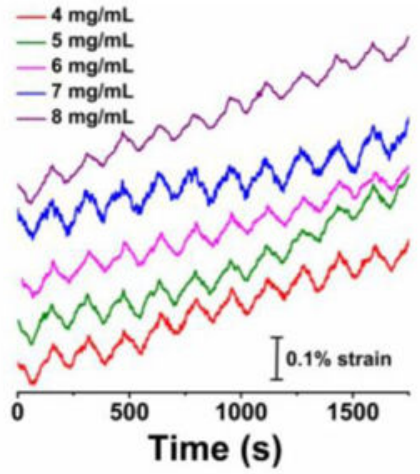

b)

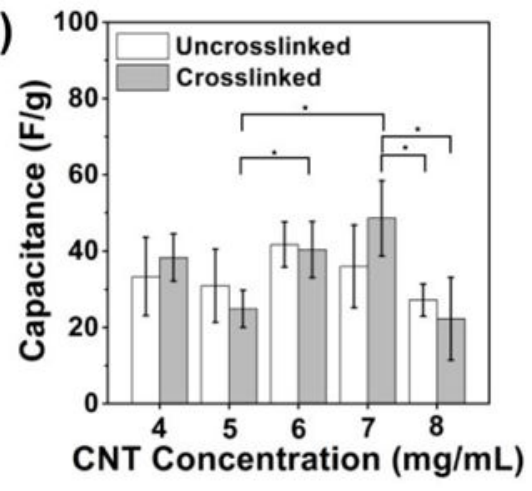

e)

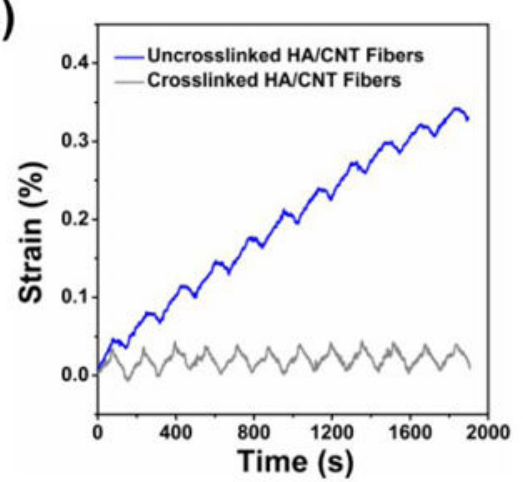

h)

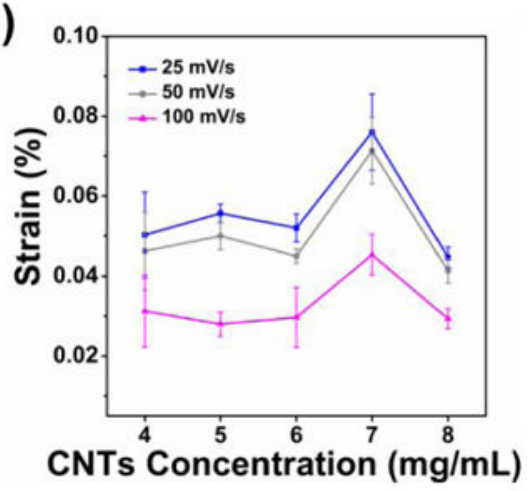

c)

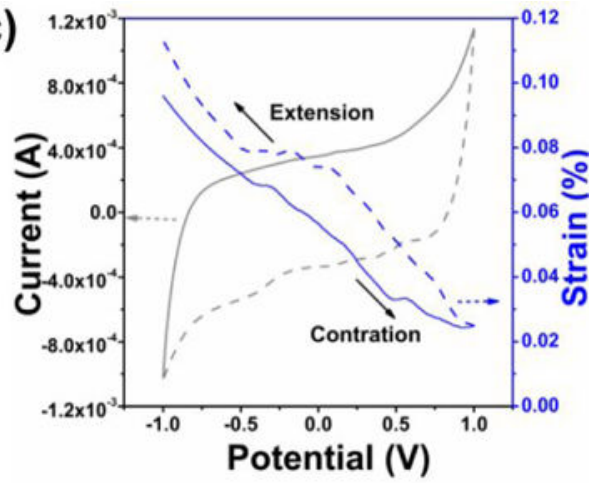

f)

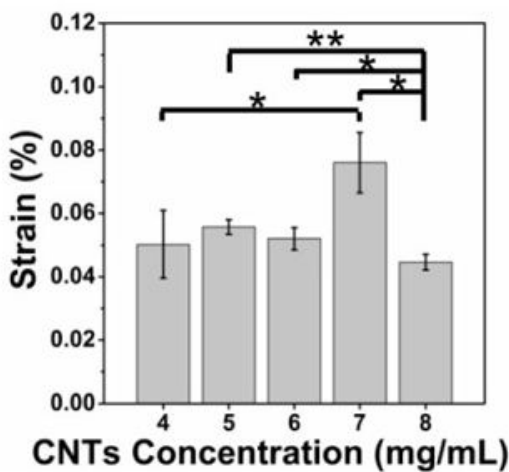

i)

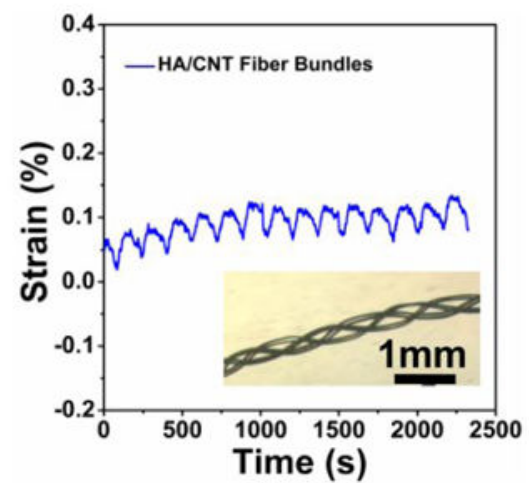

Figure 4.

$\mathrm{CV}$ and actuation behavior of HA/SWCNT microfibers. a) Typical CV curves of the crosslinked HA/SWCNT microfibers with $7 \mathrm{mg} / \mathrm{mL}$ SWCNT concentration at different scan rates in the DPBS as electrolyte. b) Electrochemical behavior of uncrosslinked and crosslinked HA/SWCNT microfibers as a function of SWCNT concentrations. c) Strain versus potential (blue color) for crosslinked HA/SWCNT microfibers with $7 \mathrm{mg} / \mathrm{mL}$ SWCNT concentration in the DPBS during cycling voltammetry (black color) (between +1 $\mathrm{V}$ and $-1 \mathrm{~V}$ versus $\mathrm{Ag} / \mathrm{AgCl}$, scan rate: $25 \mathrm{mV} / \mathrm{s}$, electrolyte: DPBS, applied load: $4 \mathrm{mN}$. The microfibers show contraction (blue solid line) and extension (blue dotted line) under oxidation (black solid line) and reduction (black dotted line) state, respectively. d) Schematic illustration of the actuation mechanism of an HA/SWCNT microfiber. e) Strain versus time for uncrosslinked and crosslinked HA/SWCNT microfibers with $7 \mathrm{mg} / \mathrm{mL}$ SWCNT concentration during CV. f) Actuation strain of crosslinked HA/SWCNT microfibers during 
cycling voltammetry as a function of SWCNT concentration. g) Actuation strain of crosslinked HA/SWCNT microfibers with different SWCNT concentrations as a function of time. h) Actuation strain of crosslinked HA/SWCNT microfibers as a function of SWCNT concentration at different scan rates. i) Actuation behavior of HA/SWCNT microfiber bundles with $7 \mathrm{mg} / \mathrm{mL}$ SWCNT concentration and an optical image in the inset. Results are presented as means \pm standard deviations $(\mathrm{n}=3 ; * p<0.05, * * p<0.01$, and $* * * p<0.001)$. 

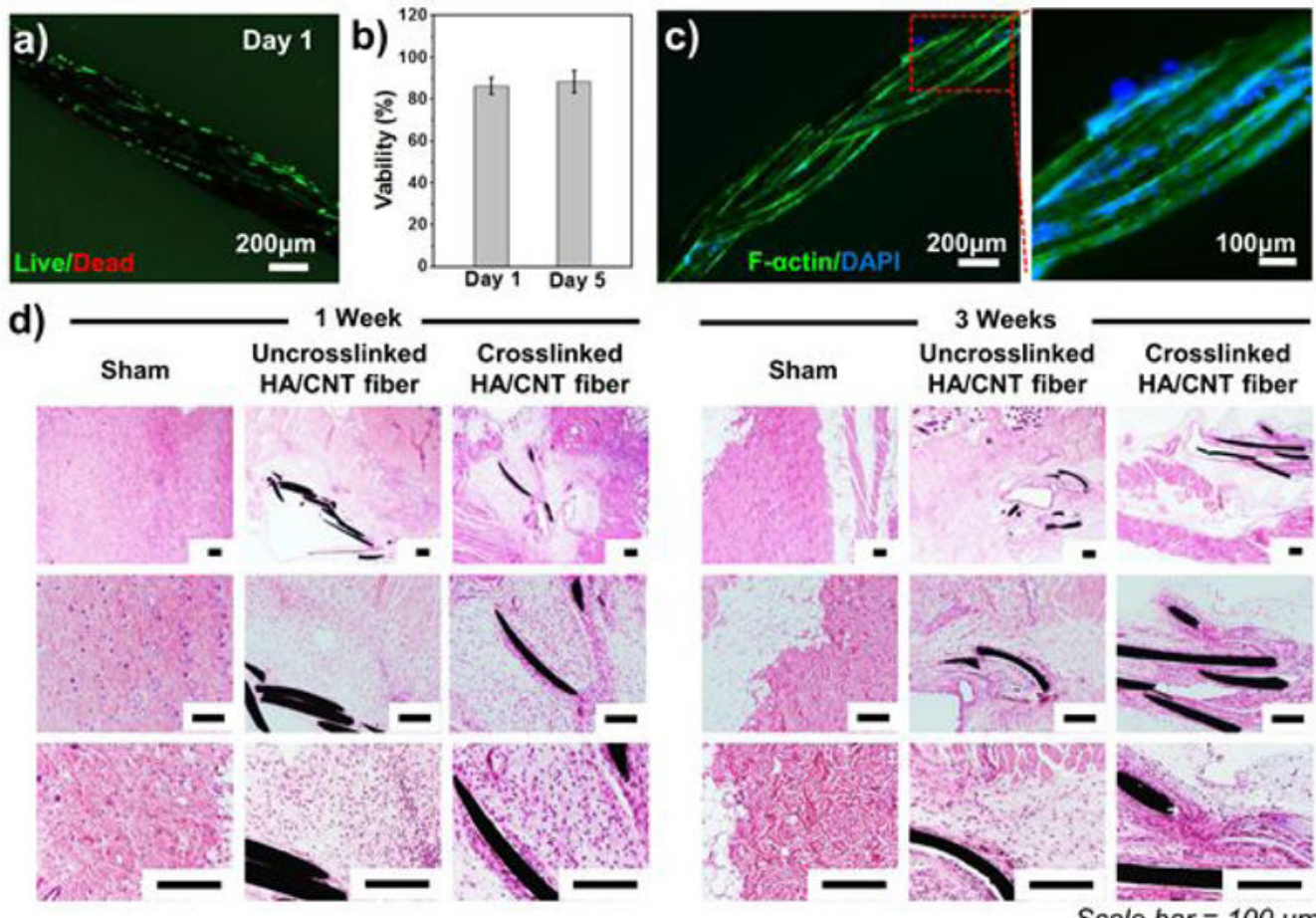

e)
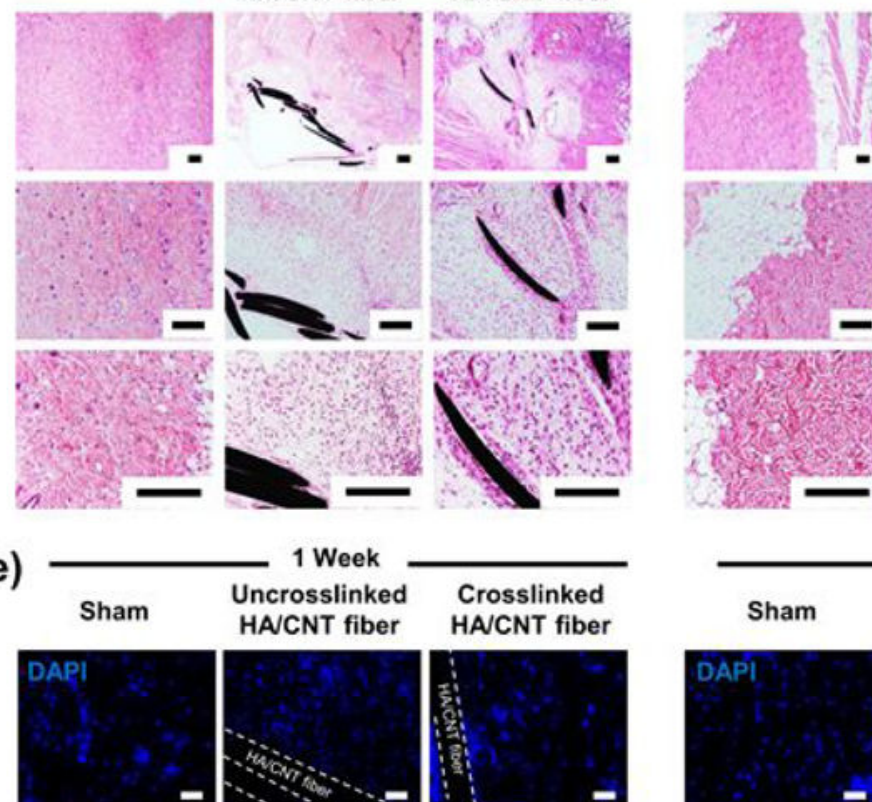

3 Weeks

Scale bar $=100 \mu \mathrm{m}$
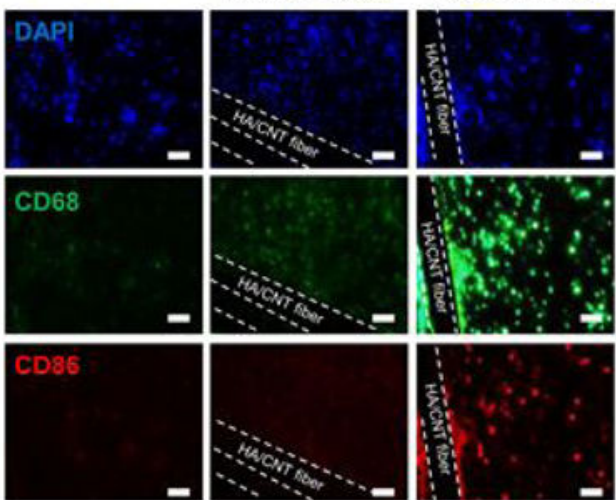

Merge
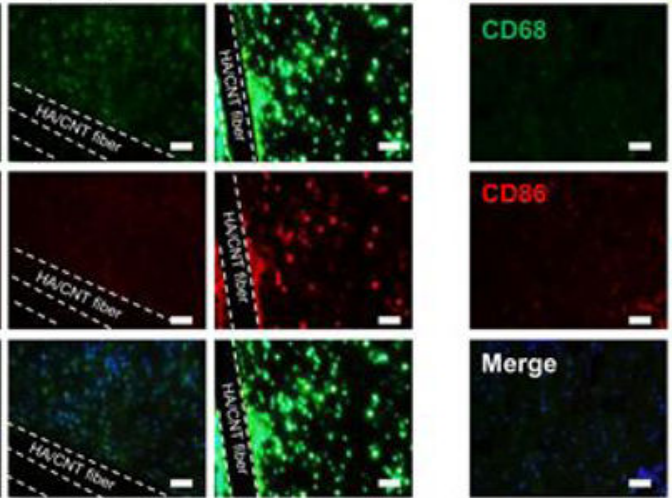

HAVNT fiber

Crosslinked
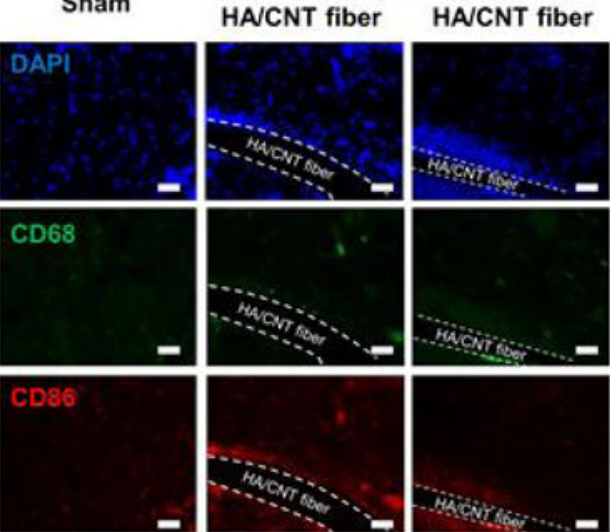

Merge

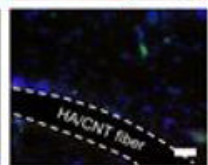

HAd
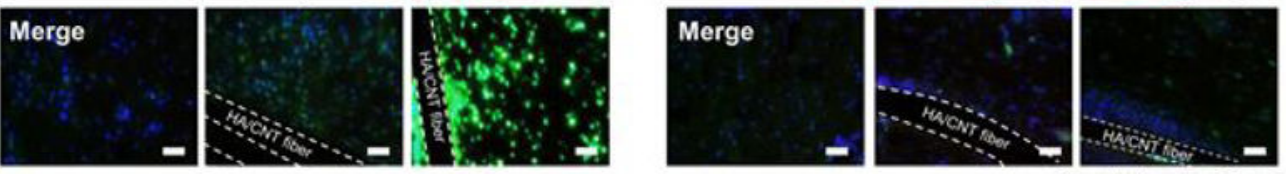

Scale bar $=50 \mu \mathrm{m}$

Figure 5.

(a, b) Viability of NIH-3T3 fibroblasts after one day and five days of culture on the surface of crosslinked HA/SWCNT microfibers with $7 \mathrm{mg} / \mathrm{mL}$ SWCNT concentration. (c)

Immunostaining of F-actin (green) and nuclei (blue) of NIH-3T3 fibroblasts after five days of culture on the surface of braided crosslinked HA/SWCNT microfibers with $7 \mathrm{mg} / \mathrm{mL}$ SWCNT concentration. (d-e) In vivo implantation of uncrosslinked and crosslinked HA/ SWCNT microfibers with $7 \mathrm{mg} / \mathrm{mL}$ SWCNT concentration in the subcutaneous tissue of mice and staining of the tissue surrounding the implanted microfibers at 1 and 3 weeks after 
implantation for biocompatibility analysis; (d) H\&E images and (e) immunohistology images stained for CD68 (monocyte/macrophage, in green) and CD86 (M1 macrophage, in red). 